\title{
Review of 20 Years of Research on Microwave and Millimeter-wave Lenses at "Instituto de Telecomunicações"
}

\author{
Carlos A. Fernandes ${ }^{1}$, Jorge R. Costa ${ }^{1,2}$, Eduardo B. Lima ${ }^{1}$, and Mário G. Silveirinha ${ }^{3}$ \\ ${ }^{1}$ Instituto de Telecomunicações, Instituto Superior Técnico, \\ University of Lisbon, 1049-001 Lisbon, Portugal \\ E-mails: carlos.fernandes@|x.it.pt; eduardo.lima@|x.it.pt \\ ${ }^{2}$ Instituto Universitário de Lisboa (ISCTE-IUL), 1649-026 Lisbon, Portugal \\ E-mail: jorge.costa@|x.it.pt \\ ${ }^{3}$ Department of Electrical Engineering, Instituto de Telecomunicações, \\ University of Coimbra, 3030 Coimbra, Portugal
}

\begin{abstract}
Starting from a challenge in the early 1990s to develop a highly shaped beam dielectric lens antenna for a pilot 150 $\mathrm{Mb} / \mathrm{s}$ cellular mobile broadband system operating in the $60-\mathrm{GHz}$ band, several new developments have been accomplished over more than 20 years at Instituto de Telecomunicações [1] in the areas of millimeter-wave shaped dielectric lens antennas and planar metamaterial lenses. We review here a few representative examples with numerical and experimental results, covering applications in mobile broadband communications, radiometry, satellite communications, multigigabit short-range communications, and sublambda near-field target detection.
\end{abstract}

Keywords: Beam-steering antennas; geometrical optics (GO) and physical optics (PO); lens antennas; metamaterial lenses; millimeter- and submillimeter-wave antennas; reflector antenna feeds; scanning antennas; shaped beam antennas; wireless communications

\section{Introduction}

D ielectric lenses were proposed as an alternative to reflector antennas as early as the 1940s [2]. Unlike reflector antennas, the beam shaping effect in lenses is achieved by placing the feed behind the lens; this eliminates the aperture blocking by the feed and the related adverse effects. Subsequent systematic studies of lens antennas showed its design flexibility to meet demanding radiation pattern specifications [3], [4]. Early lens designs were intended for microwave applications; at those wavelengths, dielectric lenses were bulky and heavy even when zoned, and thus, they were suitable only for selected applications. It was not until the 1980s and 1990s that dielectric lenses have gained new interest, pushed by new challenges in millimeter- and submillimeter-wave applications [5]-[8].

In 1992, a research team from the "Instituto de Telecomunicações" (IT) [1] participated in the European project
Mobile Broadband Systems (MBS) [9]. The project goal was to demonstrate the feasibility of a cellular mobile broadband system offering a high bit rate of $150 \mathrm{Mb} / \mathrm{s}$ per user, with mobile speeds up to $100 \mathrm{~km} / \mathrm{h}$, operating in the $60-\mathrm{GHz}$ oxygen absorption band. One of IT tasks was to develop a new antenna concept to cope with unusual specifications: due to the limited power available from solid-state devices at these wavelengths and the need to maintain multipath propagation at a controlled level, the base station antenna was required to produce constant flux illumination over the whole cell with a sharp coverage drop near the cell edges. Therefore, the antenna not only needed to produce a $\csc ^{2} \theta$ shape radiation pattern within the cell, but cumulatively the coverage footprint needed to conform to the prescribed cell geometry, typically a very elongated rectangle like a long section of a street. Highly shaped lenses with an embedded feed emerged as the preferred approach to obtain an effective relatively compact and easy to fabricate solution. Our studies have shown that embedding the feed in the lens body had added 
advantages in terms of design flexibility and lens performance for this kind of application [10]-[12]. In this case, lens design issues become different from those of classical lenses with the feed located away from the lens body [3], [5], [6].

Lens configurations with an embedded feed are usually termed integrated or substrate lens antennas [7], [8]. The concept of integrated lenses started with hemispherical lenses added on top of integrated antennas to eliminate substrate modes and increase radiation efficiency [7], [8]. This has evolved to the use of other fixed canonic shapes such as elliptical or hyperhemispherical to enhance gain, producing collimated output beams [13]. However, for more elaborate output beam specifications like those of the MBS system [10], lenses needed appropriate surface shaping. Therefore, there was a need to develop new design approaches to obtain reliable shaped integrated lens antenna for those applications [14]-[19]. The study went beyond the lens antenna development to evaluate its impact on the cell coverage and on the MBS system performance [12], [15], [19], [20].

Research on shaped lens antennas for millimeter-wave mobile communications continued at IT beyond the MBS project with other national and international research projects [17]-[22]. The same concept of shaped integrated lens antennas was later extended to millimeter and submillimeter imaging systems, in the framework of a project funded by the European Space Agency (ESA-ESTEC). The challenge was to develop lens antennas with an integrated feed to operate over $100 \%$ bandwidth, matching similar bandwidths from integrated quasi-optical receivers used in terahertz (THz) radioastronomy reflector antennas [23], [24]. Output beams were required to present Gaussissity value on the order of $90 \%$ over the band. An additional challenge was to cope with the bidirectional radiation pattern that is typical from printed feeds compatible both with $\mathrm{THz}$ fabrication technology and with the use of bolometer detectors [25], [26]. Therefore, double-shell shaped lenses with appropriate material permittivity were used to simultaneously maximize power coupling from the bidirectional feed to the lens and minimize internal reflection losses across the whole $100 \%$ bandwidth [27], [28].

Other examples of lens antennas developed at IT included the development of switched shaped beam lenses [12] as well as new compact low-cost mechanically steered lens antenna for azimuth shaped beam scanning without the need for rotary joints for high-altitude platforms [29]. This concept was later extended to include simultaneous elevation scanning, still avoiding rotary joints, intended for gigabit indoor wireless local area networks [30].

IT developed a software tool for the design, analysis, and optimization of circular symmetric shaped integrated lens antennas, complying with multiple design targets (the ILASH software tool [31]). Work on shaped integrated lenses required also a parallel effort on the design of optimal feeds for each type of lens. Other aspects of great importance were related to the selection of appropriate dielectric materials, the rigorous evaluation of the complex permittivity, the homogeneity and isotropy of these materials, and the selection of appropriate lens fabrication technology. IT contributions to these topics are also briefly addressed in this paper.

Several research laboratories, namely, from the Universities of Michigan and California Institute of Technology (CALTECH) in the USA, Siena in Italy, Eindhoven and Delft in The Netherlands, Rennes in France, and EPFL Lausanne in Switzerland, have produced landmark work on integrated lens antennas, in the majority of cases regarding canonical lens shapes such as hemispherical or elliptical [7], [8], [13], [32]-[43]. The focus was on methods to model the electromagnetic lens response, methods to design and optimize the lens performance, development of dedicated integrated feeds, and study of its influence on the lens response and lens integration with electronics. Work on lenses at IT addressed similar problems, but pioneered the study of arbitrary 3-D shaped integrated lenses and its impact on the radio channel and system performance in the context of mobile communications. The work on shaped integrated lens design was followed and immensely developed independently by the group from Rennes [44]-[46].

A natural evolution of the shaped beam lens synthesis work at IT was its extension to artificial material planar structures [47]-[49] that is still ongoing at IT. This opened a new parallel direction of research at IT dedicated to the design and characterization of artificial materials [49], [50].

This review paper is organized as follows. Sections 2 and 3 briefly discuss issues related to lens feeds and dielectric materials, respectively, and some illustrative examples are discussed. Section 4 addresses the design of shaped dielectric lenses. A few representative shaped lens configurations developed at IT are presented in Section 5. Section 6 reports the design of a planar metamaterial lens and an associated application. The conclusions are drawn in Section 7.

\section{Lens Feeds}

For any lens output specification, at least two items need to be carefully addressed prior to the lens synthesis process: the lens feeding structure and the lens dielectric materials.

Like the feeds for reflectors, lens feeds have specific requirements in terms of radiation pattern, polarization, and phase center depending on the application. This section focuses only on feeds for integrated shaped lenses, as feed design for conventional lenses (with canonical shapes) is not significantly different from feed design for reflectors. The shape of an integrated lens is tailored to obtain a prescribed output radiation pattern, and thus, it obviously depends on the feed radiation pattern inside the lens body. Three main technologies were adopted at IT for the lens integrated feeds: waveguide, coaxial, and planar printed feeds. Early examples of lens feeds developed at IT based on these configurations 

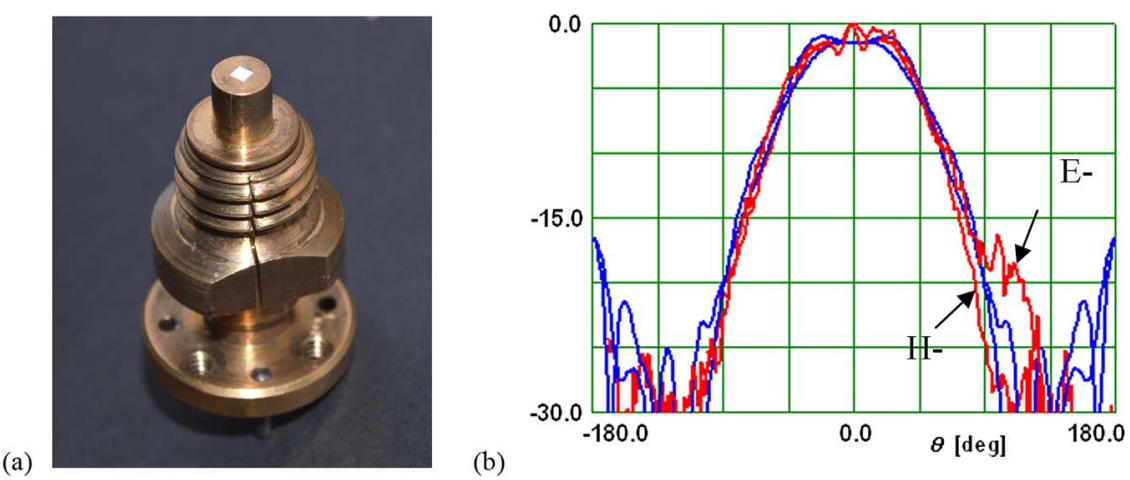

Figure 1. (a) Rectangular waveguide lens feed for the V-band. (b) Measured far-field co-polar components of the waveguide open end when radiating into MACOR (red) at $62.5 \mathrm{GHz}$, superimposed on WIPL-D simulations (blue).

were fully characterized in terms of radiation pattern and phase center in [15] and hence are not discussed here. Only the most recent advancements are reported here.

Figure 1(a) shows one example of a dedicated V-band $(50-75 \mathrm{GHz})$ waveguide feed produced at IT [51], [52] that is used in Section 5. Near its open end, the waveguide is loaded with a high permittivity dielectric wedge and tapered down to a narrow 1.4-mm square aperture designed to present a good power transfer to the lens. The square aperture produces a circularly symmetric radiation pattern inside the lens body, equivalent to a printed double-slot radiation pattern in the same conditions [13], with linear polarization and about $8 \mathrm{dBi}$ directivity (depending on lens material permittivity). This directivity is adequate to illuminate most part of the useable aperture from our shaped integrated lenses. The reflection coefficient at the waveguide port is lower than $-10 \mathrm{~dB}$.

However, some lenses have much wider bandwidth than this waveguide feed. A similar feed was fabricated for the Q-band $(30-50 \mathrm{GHz})$ with the same external dimensions, producing exactly the same symmetric radiation pattern and the same directivity [52] to allow testing wideband lenses by separate subbands.

The outer wall of the developed waveguide feeds is threaded and screwed into a matching hole opened at an extended part of the lens base. This strategy enables simple and reliable exchange of different test feeds in the same lens or the reuse of the same test feed in different lenses without compromising alignment and measurement repeatability in laboratory evaluation tests.

However, an actual ultrawideband low profile feed is also required, for example, in radio astronomy applications, compatible with simple integration of ultrawideband bolometer detectors [23], [24]. Planar antenna structures printed directly at the lens base [25], [26] are the preferred solution for submillimeter-wave applications where waveguides are difficult to manufacture. However, once the feed is printed or glued to the lens, it is not possible to reuse it in a different lens, making this type of feeds less attractive for laboratory systematic testing. A very common integrated lens feed is the double slot [13]; however, its bandwidth is limited. Wideband self-complementary log-periodic printed

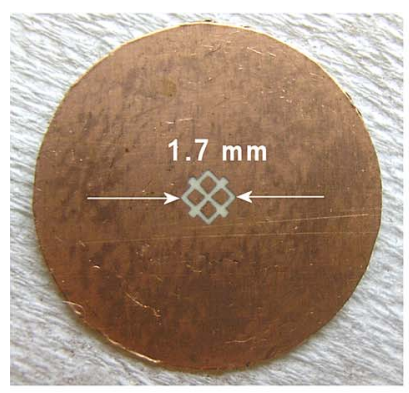

(a)

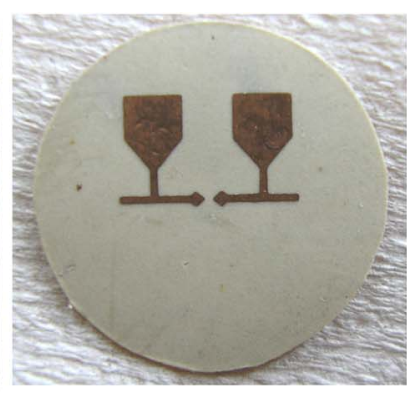

(b)

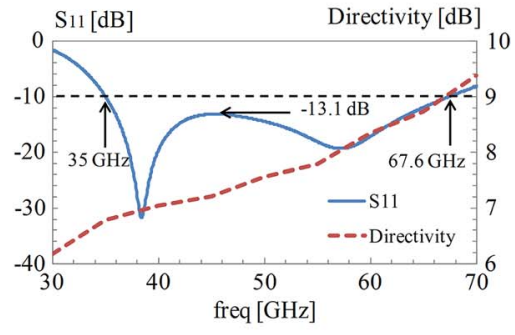

(c)

Figure 2. XETS prototype for 30-70 GHz operation when integrated with a MACOR lens. Photographs of the (a) front face and (b) back face. (c) Simulated input reflection coefficient and directivity when radiating into unbounded MACOR. 


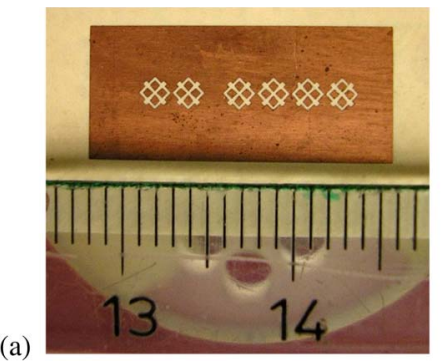

(b)

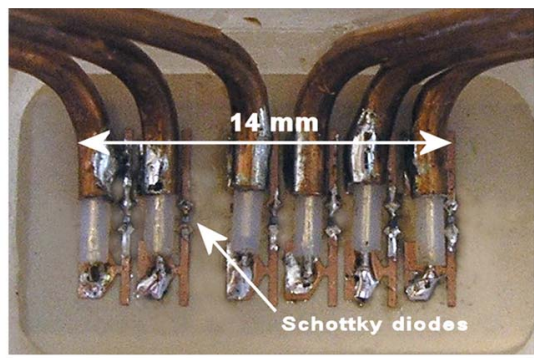

Figure 3. (a) Front side of the multiple XETS prototype close to $\mathrm{cm}$ scale, for 30-70 GHz operation. (b) Back side showing the Schottky diodes and EZ-34 coaxial cables for retrieving the IF signal.

antennas have been used as integrated lens feeds in the literature, but its polarization is not sufficiently stable versus frequency [26].

A dedicated broadband printed antenna was developed at IT, which is particularly adequate for integration with bolometers or mixer diodes. It is based on a new crossed exponentially tapered slot configuration combined with an intersecting square slot, which is onward referred to as the XETS antenna. A fabricated prototype for $30-70 \mathrm{GHz}$ operation attached to a MACOR lens [53] is shown in Figure 2. The feed is developed in two layers of metallization [53]. The slot layer is in direct contact with the lens base; the other layer [see Figure 2(b)] contains a replica of two opposing central petals from the front face, printed at the same position in order to provide a capacitive coupling with the front layer. These petals are used to mount a Schottky diode (used as a mixer). The intermediate frequency (IF) retrieving circuit with soldering pads for the coaxial cable is also shown in Figure 2(b). The local oscillator (LO) signal is radiated through the air into the circuit, as will be explained in Section 6.2. Depending on the lens permittivity, more than $60 \%$ impedance bandwidth can be achieved [see Figure 2(c)] with reasonably stable radiation pattern and almost pure linear polarization across the whole bandwidth; the cross-polarization level is on the order of $-15 \mathrm{~dB}$. This is a clear advantage over self-complementary logperiodic printed feeds [26]. The phase center of this radiating element is very stable across the whole frequency band and lies in the antenna center.

These broadband XETS elements can be closely packed for scanning lens applications [see Figure 3]. The multiple XETS elements are aligned along the feed H-plane [54]. Simulations have shown that a separation between XETS centers of only $2 \mathrm{~mm}$ in this plane ensures better than $18 \mathrm{~dB}$ isolation between adjacent feeds across the operating bandwidth, despite the presence of the very close IF circuits on the back face.

\section{Dielectric Materials for Lenses}

The second issue to be addressed prior to the lens design is the selection and characterization of the dielectric material.
Common materials are polyterafluorethylene, polystyrene, fused quartz, ceramics, silicon, or alumina. In most cases, the electromagnetic characteristics of candidate materials are specified by the manufacturer for microwave frequencies only, with tolerances that are incompatible with an accurate lens design in the most stringent applications. Thus, the dielectric complex permittivity must be evaluated in-house to ensure a good agreement between predictions and measurements. In addition to the electrical properties, the homogeneity and the isotropy of the raw material must also be checked.

Two classical methods are used at IT for complex permittivity measurement: the waveguide method [55] and the open resonator Fabry-Pérot method [56], [57]. The waveguide method is very convenient for low to moderate permittivity and losses ( $\varepsilon_{r}$ up to 5 and $\tan \delta$ in the $10^{-4}-10^{-2}$ range). Parallelepiped samples of the dielectric material are precisely cut to fill completely the cross section of the rectangular waveguide short-circuited sample holder. Under single-mode $T E_{10}$ conditions, the complex permittivity is calculated from the measured $s_{11}$ frequency responses for the 1) dielectric filled and 2) empty sample holders. The method is quite accurate, provided that the air gaps between the sample and waveguide walls are less than $50 \mu \mathrm{m}$ in the V-band. Because the required samples are very small (typically $3.9 \times 1.9 \times 5.0 \mathrm{~mm}^{3}$ in the V-band), material nonhomogeneity or anisotropy can be evaluated by cutting samples from different parts and different orientations of the raw material block.

Excitation of higher order modes in the dielectric filled sample holder for relative permittivity values typically larger than 5 tends to introduce significant error in the complex permittivity determination, particularly in the loss tangent. The FabryPérot open resonator method [56] does not have this limitation. A plane-concave configuration was selected since it involves the fabrication of only one spherical mirror while allowing precise positioning of the dielectric samples directly on the planar mirror, without any perturbation from sample holders [see Figure 4(a)]. The resonator dimensions were chosen to produce at the planar mirror a $6-\mathrm{mm}$ beam waist fundamental Gaussian mode with well-defined linear polarization. The quality factor of the empty Fabry-Pérot resonator is $10^{6}$. The complex permittivity of the sample dielectric material disk is calculated from the $s_{21}$ frequency response measured between 
the feeding and pick-up waveguide probes [57] for the empty and for the dielectric loaded resonator. The measurement is sensitive to polarization, and thus, by rotating the sample disk about the cavity $z$-axis, it is possible to clearly identify possible anisotropies of the material sample and calculate the complex permittivity along the anisotropy axes [57].

The measured permittivity for typical dielectrics is shown in Figure 4(b). High permittivity values of the lens material allow more flexibility to comply with beam shaping requirements and better power coupling from bidirectional planar feeds to the lens. However, this comes at the cost of higher lens internal reflection loss and detuning of the feed input impedance [37]. Matching layers [40] or multiple shell lenses can cope with this opposite dependence on material permittivity.

In addition to the electromagnetic properties, the choice of the dielectric material may be limited by the selected process for lens fabrication. Milling is a common approach, but it may not be appropriate for hard materials, typically with high permittivity values such as alumina. In [46], a layer deposition method is used for alumina.

Concerning metamaterial lenses, our studies were focused on planar configurations formed by stacking numerous layers of ROGERS duroid substrate [58], each one with an adequate printed metallization pattern. This allows tailoring the effective index of the bulk medium to produce prescribed nonuniform index profiles or to produce a uniform very high refractive index medium in a controlled way. Determination of the effective permittivity in the first case can be made directly using the waveguide method under certain conditions [49] or indirectly in the second case by analyzing the wavenumber in a guiding structure formed by this material.

\section{Design of Shaped Dielectric Lenses}

Figure 5 presents the generic lens geometry that has been addressed at IT. It consists of a single- or double-shell

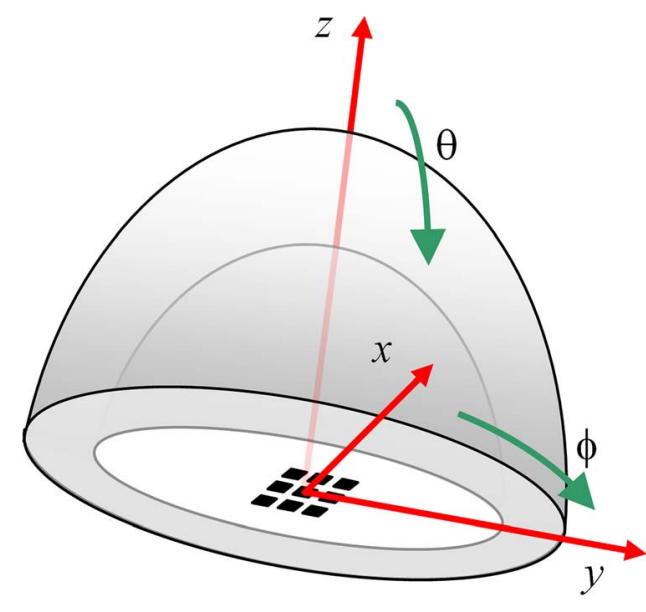

Figure 5. Generic geometry of shaped lens antennas developed at IT.

dielectric lens, shaped in the elevation coordinate $\theta$ and eventually also in azimuth coordinate $\phi$. It may be fed by a single feed or by an array of feed elements attached or not to the lens base. In the general case, feed elements are either on-axis $(x=0 ; y=0 ; z=0)$ or off-axis. Different options exist for the feed element, as discussed in the previous section, and of course, its configuration and radiation characteristics influence the final lens shape.

The adopted design procedure for shaped lenses is summarized in Figure 6. Two alternative approaches are presented: one provides a fast first guess of the lens shape, based on closed-form analytical expressions-labeled a); the second more elaborate approach—labeled b)—combines lens performance analysis tools with an optimization algorithm that successively generates and evaluates meaningful populations of test lenses until a specified figure of merit is reached. Both approaches were adopted in the ILASH lens antenna design tool developed at IT [31]. ILASH is restricted to axial-symmetric lenses with canonic or shaped elevation profile ( $\theta$ coordinate). The details of the design steps are described in the following sections.

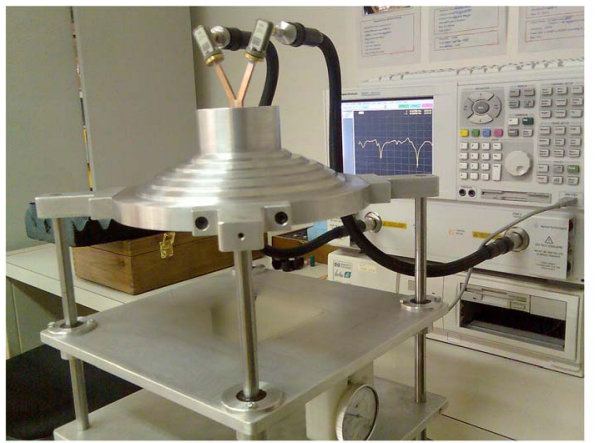

(a)

\begin{tabular}{|c|c|c|}
\hline Material & $\boldsymbol{\varepsilon}_{\mathbf{r}}$ & $\tan \delta$ \\
\hline Eccostock K1.7 & 1.7 & 0.005 \\
\hline Polystyrene & 2.55 & 0.002 \\
\hline Fused quartz & 3.82 & 0.0015 \\
\hline PLEXYGLAS & 2.53 & 0.012 \\
\hline MACOR & 5.5 & 0.0118 \\
\hline Alumina & 9.4 & 0.0013 \\
\hline
\end{tabular}

(b)

Figure 4. (a) Fabricated Fabry-Pérot open resonator for V-band complex permittivity measurements. (b) Measured permittivity values of different materials at $62.5 \mathrm{GHz}$. 


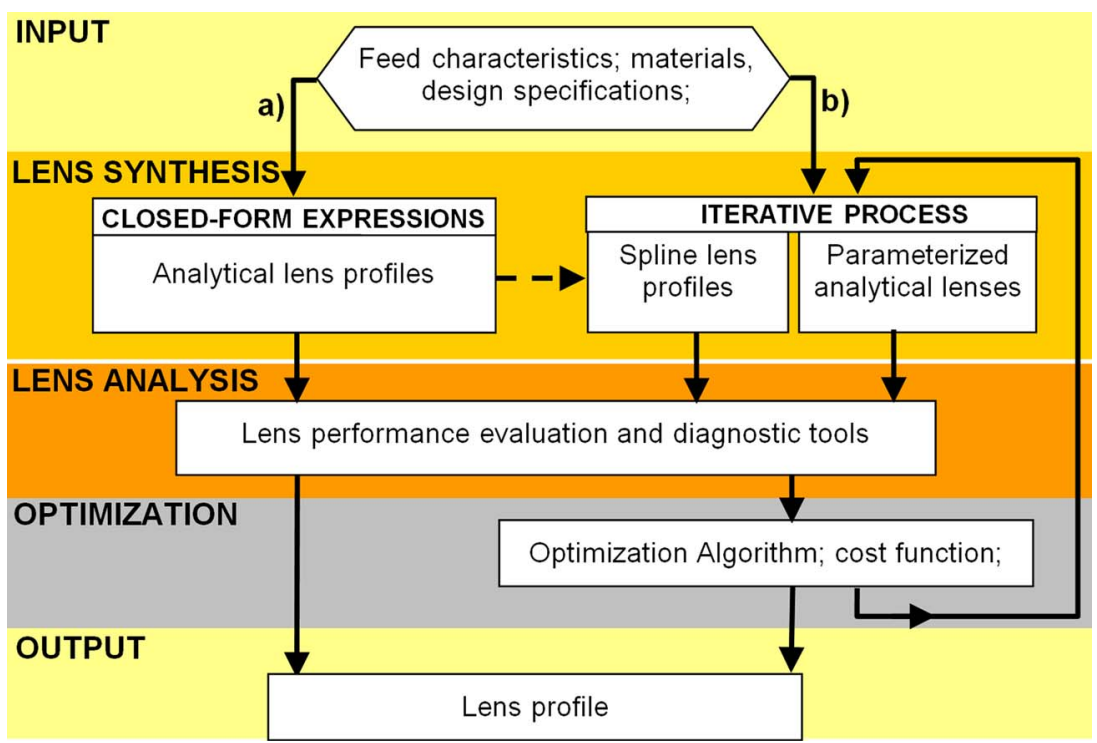

Figure 6. Block diagram of lens design steps.

\subsection{Direct Lens Solutions}

Closed-form lens synthesis expressions can be obtained as a first guess for some classes of target specifications, based upon geometrical optics (GO) formulation. The lens overall dimensions and the principal radii of curvature at every point on the surface are required to be much larger than the wavelength to ensure the GO validity. The feed is characterized only by its phase center and by its far-field pattern in an unbounded medium with the same permittivity of the lens material. The GO lens designs are inherently broadband as long as the lens dimensions are large compared to the wavelength, the material dispersion is negligible, and the feed radiation pattern remains constant versus frequency.

The lens shape is directly determined from the solution of a set of partial differential equations corresponding to
Snell laws at the lens interfaces and additional conditions related to the output requirements. The calculation time is generally on the order of seconds, which makes it attractive as a first guess and as a seed for a subsequent refinement optimization loop if required. Alternatively, the GO formulation can include shaping parameters that enable running the closed-form expressions in the optimization loop b) of Figure 6 to match an appropriate cost function [31]. This narrows down the search space to physically meaningful lenses.

Analytical formulations for shaped lens design were developed for different output specifications and lens system configurations (see Table 1). Earlier designs involved a single output condition - fixed beam complying with a far-field output template (the target far-field radiation pattern specification). This requires just a single lens refracting surface. Both axis-symmetric [11], [12] and generic 3-D lenses were

Table 1. Summary of All Analytical Formulations Developed at IT Based on GO for Shaped Lens Design.

\begin{tabular}{|c|c|c|c|c|c|}
\hline \multirow{3}{*}{\multicolumn{2}{|c|}{ Output beam requirements }} & \multicolumn{4}{|c|}{ Lens system configuration } \\
\hline & & \multirow{2}{*}{ Feeding } & \multicolumn{2}{|c|}{ Single Shell } & \multirow{2}{*}{$\begin{array}{c}\text { 2-Shell } \\
\text { BOR } \\
\end{array}$} \\
\hline & & & BOR & 3D & \\
\hline \multirow{3}{*}{ 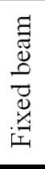 } & Far-field template & \multirow{3}{*}{ Single-feed, on-axis } & {$[12],[1]$} & [14], [19] & \\
\hline & $\begin{array}{l}\text { Far-field template }+ \\
\text { maximum power transfer }\end{array}$ & & & & {$[22]$} \\
\hline & $\begin{array}{c}\text { Far-field template }+ \\
\text { phase center position }\end{array}$ & & & & {$[28]$} \\
\hline \multirow{4}{*}{ 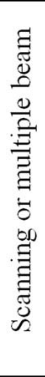 } & $\begin{array}{l}\text { Abbe sine condition }+ \\
\text { collimated beam }\end{array}$ & $\begin{array}{l}\text { Multi-feed, stand- } \\
\text { alone lens }\end{array}$ & & & {$[60],[61$} \\
\hline & $\begin{array}{l}\text { Abbe sine condition }+ \\
\text { Phase center position }\end{array}$ & $\begin{array}{l}\text { Multi-feed and lens } \\
\text { plus reflector }\end{array}$ & & & {$[62]$} \\
\hline & $\begin{array}{l}\text { Far-field template in } \\
\text { elevation + collimated } \\
\text { beam in azimuth }\end{array}$ & $\begin{array}{c}\text { Single feed, off-axis, } \\
\text { mechanical steering } \\
\text { in azimuth }\end{array}$ & [29] & & \\
\hline & $\begin{array}{l}\text { Collimated beam }+ \\
\text { maximum scanning angle }\end{array}$ & $\begin{array}{c}\text { Single feed, } \\
\text { mechanical steering } \\
\text { in azimuth and in } \\
\text { elevation }\end{array}$ & & & {$[30]$} \\
\hline
\end{tabular}


developed [14], [19] to produce $\sec ^{2}$-type elevation radiation patterns, in some cases also with shaping in azimuth. Later on, expressions were presented also for nonuniform refractive index planar lenses based on artificial dielectric media [47][49]. By adding a second shell to a lens, it is possible to impose an additional design specification. Formulations were presented for double-shell axis-symmetric lenses combining a far-field template with a maximum power transfer condition [22] or alternatively with the specification of a well-defined phase center position [28]. These lenses were studied as high Gaussissity feeds for reflectors.

Integrated lenses are also attractive for scanning or multibeam applications at millimeter waves and THz. They can be glued directly on top of an array of feeds on chip to form a compact stand-alone multibeam antenna. GO-based formulations were developed for double-shell axial-symmetric integrated lenses imposing simultaneously a reduced phase aberration condition for off-axis feed positions and a ray collimation condition for the beams [59], [60]. These lenses can scan up to 5 beamwidths with less than $1.5 \mathrm{~dB}$ gain scan loss. Integrated scanning lenses can be combined with a reflector to significantly increase the output gain. In this case, the beam collimation condition was replaced by another one imposing a well-defined phase center position lying far behind the lens body and coincident with the focus of the reflector [28]. This concept has been explored to produce multiple virtual foci in the reflector focal plane using a single lens [61], which is attractive to increase the number of beams using less space than conventional focal plane arrays.

A new compact and simple mechanically steered beam concept has also been developed, starting with a closed-form formulation. The beam scanning is obtained by mechanically moving a specially shaped lens in front of its stationary feed, without touching it [29], [30]. This avoids the otherwise need for fault-prone rotary joints in long continuous usage.

A few practical examples from Table 1 are presented in more detail in Section 6.

\subsection{Shaped Lens Analysis and Optimization}

The GO-based formulation discussed in the previous section is inherently independent from frequency. An appropriate analysis method is required to evaluate the lens actual performance taking into account diffraction effects, frequency dependence of the feed radiation pattern, and material dispersion.

The two-step GO/physical optics (PO) method [13] is widely used in the literature and adopted at IT to determine the radiation pattern of lens antennas (also of reflector and other aperture antennas). It offers a very good compromise between quality of results and computation time, making it attractive for integration into lens design optimization loops. It takes as input the lens shape, the material permittivity, the feed position, and the far-field pattern from the feed in the lens material. The GO is used for evaluating the field distribution over the lens/reflector surface, and PO is used to calculate its contribution to the near- or far-field radiation. $\mathrm{GO} / \mathrm{PO}$ is particularly useful for very large structures in terms of wavelength, and there is no upper size limit for its applicability. On the contrary, GO fails for small lenses [62], where the feed can no longer be replaced by its far-field radiation pattern and the ray propagation approach is no longer valid.

An alternative formulation was developed at IT to circumvent some of the indicated limitations of $\mathrm{GO} / \mathrm{PO}$ in the characterization of small lenses [63]. It is a PO-based method that uses point-like sources as basis functions to decompose the precalculated feed aperture fields at the lens base and calculate the resulting incident near field at the lens surface. The proposed method was implemented only for axial-symmetric lenses with on-axis feed.

Another limitation of the GO approach is that it ignores possible surface waves that can be excited at the lens radiating interface [38]. However, our analysis showed that this effect is more important in collimating lenses with canonical shapes, such as the elliptical lens; it is imperceptible in most of the shaped lenses designed at IT.

Full-wave numerical solvers such as the CST Microwave Studio transient solver [64] were used at IT to analyze the final lens design prior to fabrication. Such tool takes into account all the effects that are handled with some approximations in the previously discussed methods, but due to the involved computation time, it is not viable option for integration in lens design optimization loops.

The genetic algorithms (GA) optimization method has long been used at IT [48] because of its flexibility, robustness, global search character, inherent capability to avoid trapping in local minima, and complete absence of restrictions on the behavior of the function to be optimized. The drawback is its relatively slow convergence, and hence, two different approaches were used to reduce the number of shape parameters that model the unknown lens surfaces (parallel boxes in the b) path of Figure 6):

- $\quad$ spline-based representation of the lens surface;

- parametric optimization of selected parameters from the GO closed-form design expressions.

The spline-based representation is the most flexible of the two solutions, allowing for a limitless search space. As few as 4 to 5 nodal points $c_{n}$ of the lens surface are used as the target for optimization [see Figure 7], whereas a cubic spline approximation is used to represent the lens surface between consecutive pairs of these wide-spaced nodal points. Particularly with double-shell lenses, this strategy often leads to the presence of caustics near the outer lens surface. These lenses are automatically eliminated from the test population, but this may significantly reduce the number of "individuals" 


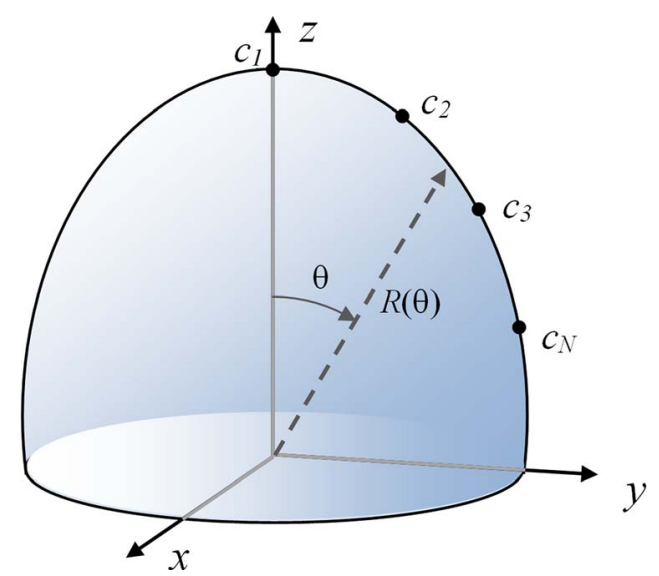

Figure 7. Geometry for spline parameterization of a generic lens interface.

in the test population, with a negative impact on the convergence of the optimization method.

The alternative strategy was to construct the test population based on lens shapes obtained directly from the previously described GO closed-form expressions, where the shaping parameters are incorporated. These shaping parameters were used as the target for optimization, thus ensuring that all the generated lenses are necessarily viable and none needs to be eliminated a priori. This favors convergence.

In order to quantify the merit of a lens in the optimization procedure, versatile cost functions were defined combining different target conditions such as far-field amplitude template, maximum power transfer, main beam power transfer, Gaussissity, phase center position, or beam scanning. For broadband and for scanning lens design, multifrequency and/or multifeed position optimization runs were required.

A software tool was developed at IT (ILASH) [31] that implements the previously described methods for the design, analysis, and optimization of axial-symmetric multishell lens antennas with a homogenous refractive index. Its interface, as presented in Figure 8, provides the user with a simple way to interact with the kernel, to generate and manipulate lens design data, to fully characterize the lens performance, and to export and import results. It is also possible to monitor in real time several aspects of the optimization process [see Figure 9], such as the cost function value, lens parameter evolution, and convergence.

\section{Design of Metamaterial Lenses}

Planar lens configurations are attractive because they enable the use of low-cost printed circuit fabrication techniques and easy integration with planar circuit devices. In this case, beam shaping requires adequate profiling of the lens permittivity, where rays continuously bend from the source to the lens output surface, to produce a prescribed output beam [47]. Artificial media are the most viable approach for obtaining a profiled material permittivity. Such materials are formed by a host dielectric medium loaded with dielectric or metallic inclusions (small compared to wavelength) so that the macroscopic behavior is similar to a continuous dielectric material characterized by effective constitutive relations. In some cases, the macroscopic response of these materials may be rather different from that of conventional natural media. These are called metamaterials. Different types of inclusions were analyzed at IT for the synthesis of these artificial materials, namely, conductive disks or planar wires (strips), which are compatible with printed circuit technology. Due to the shape of the inclusions and because of the limitations of a planar design, the permittivity of the resulting bulk material is anisotropic, and this effect has to be accounted for in the lens design.

Work on metamaterial lenses at IT went beyond shaped beam lenses and has been directed to near-field imaging

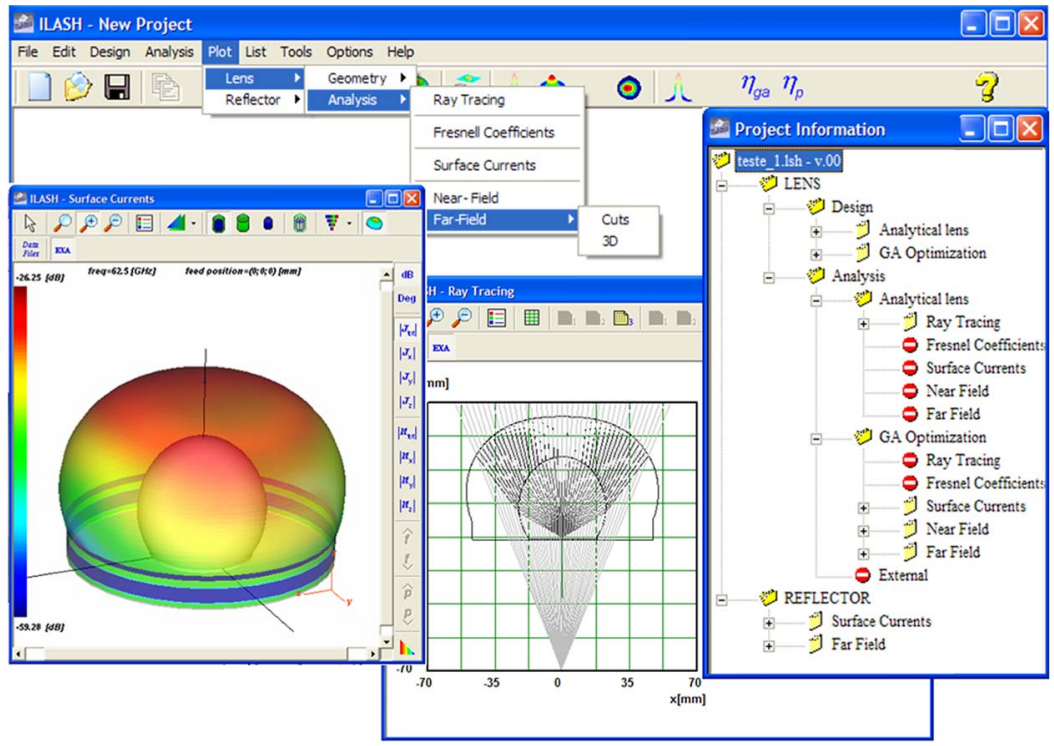

Figure 8. Screenshot of the ILASH software tool [31]. 


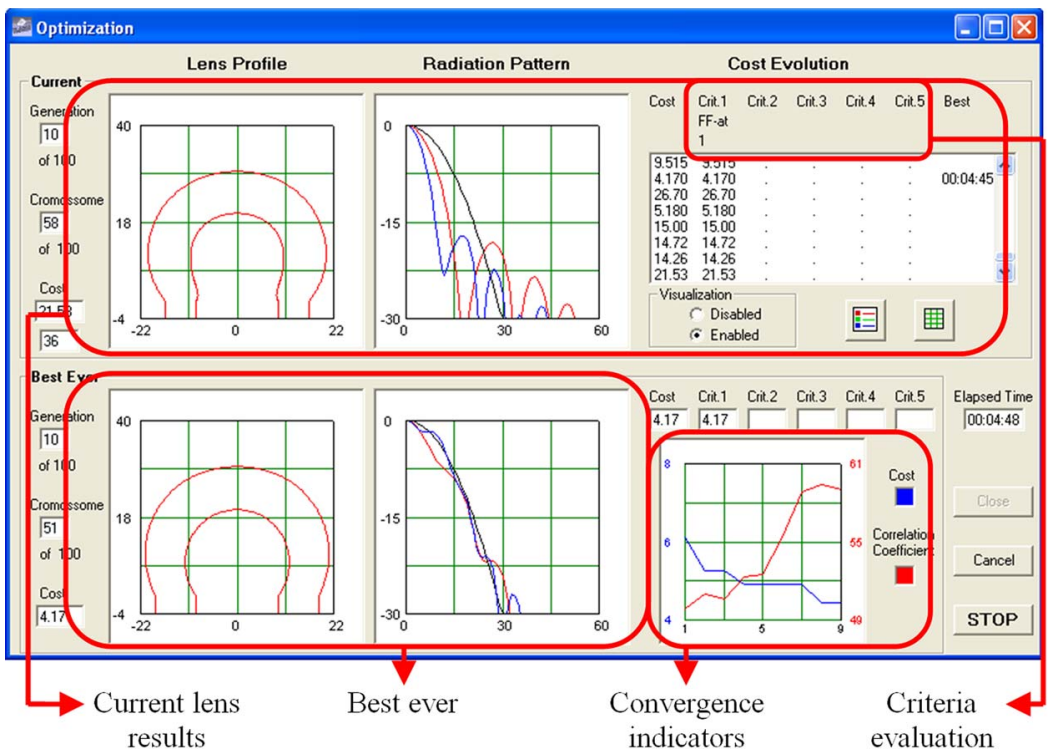

Figure 9. GA optimization monitoring screen [31].

lenses as well. With this purpose, much work was devoted to the characterization of a new medium consisting of an ultradense array of finite-length nonconnected crossed metallic wires - the crossed-wire media [50]. It was shown that a slab made of this material could present extreme values of the effective refraction index and support tightly bound surface wave modes, with very fast field decay in the transversal plane. These near-field evanescent waves can be used to restore the fine details of an object in the vicinity of the lens, beating the Rayleigh $\lambda / 2$ diffraction limit [65], [66]. Although this topic of superlensing is widely addressed in the literature, our work brought important new features that are described in one of the examples from Section 6.

\subsection{Case of Shaped Beam Lenses}

The design of shaped beam metamaterial lenses at IT was restricted to axis-symmetric cases with a single output condition specification: the far-field amplitude template. Due to the axial symmetry, the problem could be solved in a single cross section of the lens. The design procedure comprises two main steps: one is related to the determination of the permittivity profile, assumed as a continuous function of space coordinates; the second step corresponds to the determination of the spatial distribution of inclusion sizes that are required to match the prescribed permittivity profile [see Figure 10].

The lens medium is described by a coordinate-dependent permittivity tensor reflecting the anisotropy that is due to the shape and orientation of the selected inclusion. The GO formulation [67] is used to calculate the required spatial profile of the permittivity. As aforementioned, because of the selected inclusions and of the adopted layout [47]-[49], the resulting medium is uniaxial. Thus, with appropriate feed polarization, the unknown reduces to a single element of the permittivity tensor, and it only depends on a single coordinate.

A system of differential equations obtained from a suitable Hamiltonian [67] describes the ray trajectory as a function of the unknown permittivity profile. An additional differential equation establishes the relation, which depends on the unknown permittivity profile, between the output
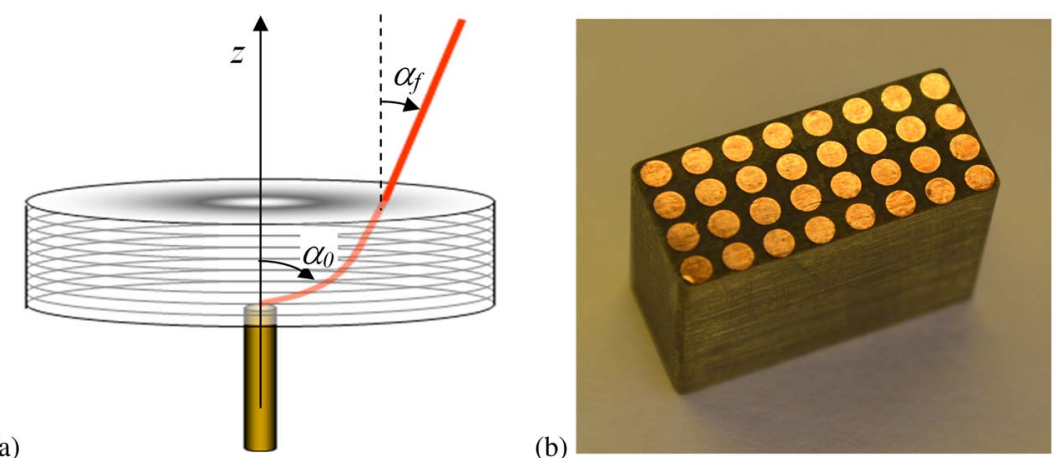

Figure 10. (a) Geometry of planar lens formed by stacking printed circuit boards. (b) Sample of disk media for measurement of effective permittivity. 
angle $\alpha_{f}$ of each ray trajectory and the corresponding shooting angle $\alpha_{0}$ that is required to transform the feed power pattern to the prescribed output far-field power template. The permittivity function is then inverted in closed form or numerically to satisfy simultaneously the pertinent differential equations [47]. Alternatively, the inversion step can be replaced by an optimization loop where the unknown permittivity profile is represented by a polynomial function and its coefficients are then optimized in a loop, solving repeatedly the direct problem until a satisfactory solution is found [48].

Once the permittivity profile is known, the required material must be synthesized. The effective permittivity of the artificial medium depends on the volume fraction that is filled with the inclusions. The relation between the effective permittivity and the size of the particles has been calculated by many authors for different types of inclusions [68].

\subsection{Near-Field Imaging Lens}

As previously mentioned, a crossed-wire metamaterial slab was used at IT for microwave near-field imaging. In general terms, this metamaterial can be regarded as an anisotropic dielectric slab waveguide, characterized by homogenized material constitutive parameters. However, due to the fact that the inclusions (continuous metallic wires) are spanned over many unit cells of the material, classical homogenization methods for unbounded bulk media are not valid for these structures [50], and classical boundary conditions are incomplete to describe the fields at the interfaces. The authors have demonstrated a new modified homogenization procedure with modified boundary conditions that can accurately characterize the reflection and transmission of waves by thin metamaterial slabs, remaining valid for wide incident angles and for evanescent waves [69]. Interestingly, the new approach requires just the knowledge of the unbounded bulk medium material properties but adds accuracy to the description of wave interaction with metamaterial slabs of arbitrary thickness.

After the transmission coefficient through the slab is obtained for a plane incident wave, the structure parameters (slab thickness and wire lattice constant) are optimized to enhance the excitation of guided evanescent waves while minimizing the excitation of guided propagating waves. While image resolution based on propagating waves is bounded by the $\lambda / 2$ diffraction limit, evanescent waves can retain sublambda details in the near field. Since the size of the wires is much smaller than the wavelength, the effect is nonresonant, and it holds for a reasonable bandwidth.

\section{Examples of Developed Lenses}

This section presents selected lens examples out from a large collection developed over the years at IT for different applications. These and other examples are discussed in full detail by the authors in the indicated references.

\subsection{Shaped Lens for Wireless Mobile Broadband Communications}

The cellular MBS concept was introduced in 1992 and developed throughout the 1990s [9]. It was intended to transport, in a transparent way, asynchronous transfer mode cells over the air interface at data rates up to $155 \mathrm{Mb} / \mathrm{s}$, withstanding mobile velocities up to $100 \mathrm{~km} / \mathrm{h}$, while maintaining the quality of service of the fixed network. Provisional subbands were allocated for these systems at 39.5-40.5, 42.5-43.5 $\mathrm{GHz}$ and 62-63, 65-66 GHz.

At the base station, a shaped beam was required to approximate a constant flux illumination of the cell to favor the link budget, with a reasonably sharp drop at the cell edge to control cell spillover [see Figure 11(a)]. This characteristic corresponds to $\csc ^{2} \theta$-type gain contribution from the antennas to the link budget within the cell, which ideally compensates for the free-space loss at each observation direction. An acrylic 3-D lens was designed using a modification of GO closed-form expressions to produce constant flux illumination across a long street section when hanging from a lamp post located $10 \mathrm{~m}$ above the street level at the center of the cell [21]. The lens was fed by the embedded open-end aperture of a dielectric loaded rectangular waveguide operating in the $T E_{10}$ mode. The lens prototype is shown in Figure 11(b) [10], [21]. The dimensions are $10 \times 4 \times 2 \mathrm{~cm}^{3}$, and the lens produces a bidirectional radiation pattern with $13.2 \mathrm{dBi}$ gain at $62.5 \mathrm{GHz}$ at the maximum distance direction $\left(\theta_{\max }, \varphi= \pm 90^{\circ}\right)$, with linear polarization [10]. The lens was successfully used in an outdoor system demonstrator operating at a $32 \mathrm{Mb} / \mathrm{s}$ gross bit rate [16]. In a follow-up project, these lenses were redesigned for the $40-\mathrm{GHz}$ band with the same specification but using a lower loss dielectric material, i.e., polystyrene [15]. A similar radiation pattern was obtained in this case, but with slightly higher gain of $14 \mathrm{dBi}$. The received power over the street cell is plotted in Figure 11(c). It shows that by using a single shaped lens antenna at the center of the cell, it is possible to produce a rectangular type of coverage above the threshold level, almost confined to the cell limits. No similar type of performance has been presented before for any antenna.

In a different configuration, a 3-D lens was developed to produce a constant flux illumination with sharp square boundaries, appropriate for indoor room coverage with controlled wall illumination, as shown in Figure 12(a) [19]. The lens was made of acrylic with the size $84 \times 32 \mathrm{~mm}^{2}$ (diagonal $\times$ depth) for operation at $62.5 \mathrm{GHz}$. The lens was fed by the circularly polarized $T E_{11}$ mode of a circular waveguide immersed in the lens body and mounted near the ceiling. Figure 12(b) shows the received power distribution beneath the lens, measured while the receiving lens antenna, with a flat-topped radiation pattern [15], traveled on a kart to scan a 


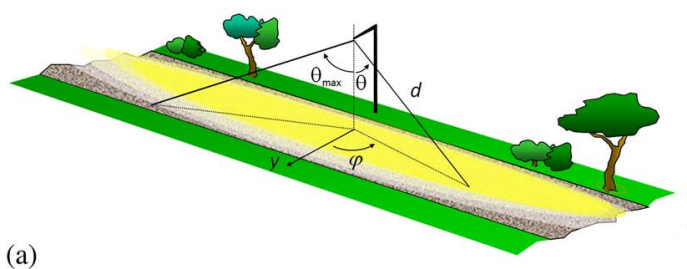

(b)

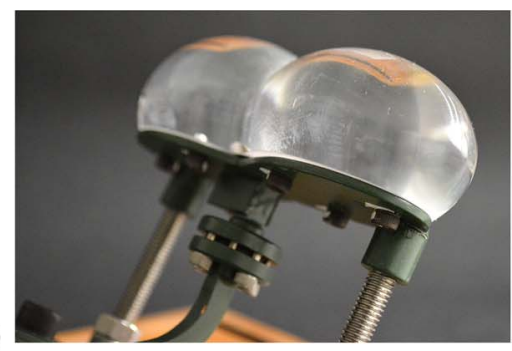

Normalised Received Power
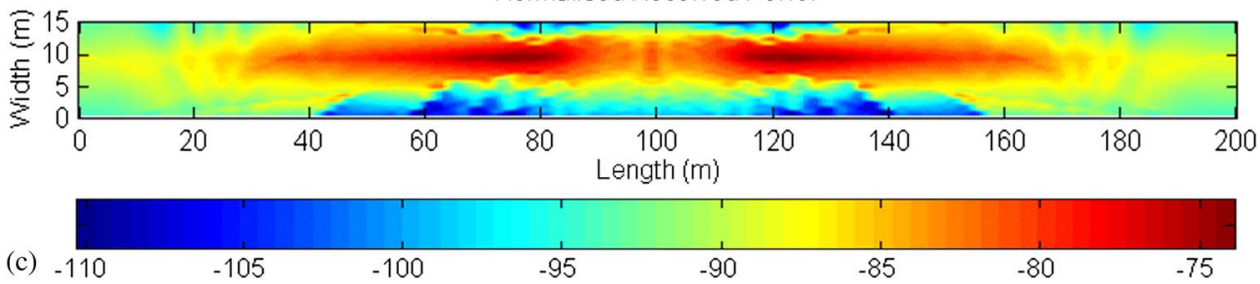

Figure 11. (a) Geometry of a street sectored cell. (b) Photograph of a shaped lens antenna for a constant flux illumination of the street at $60 \mathrm{GHz}$. (c) Received power within the cell limits for a transmitted power of $22 \mathrm{dBm}$.

$4 \times 8 \mathrm{~m}^{2}$ floor area. The difference in height between the base station and the mobile terminal antenna was $0.5 \mathrm{~m}$. The figure shows a constant received power in the illuminated region and a sharp drop at the cell edge as required. The $\mathrm{sec}^{2}$ radiation pattern has the interesting property that the cell dimensions can be scaled just by changing the height difference between the fixed and the mobile antennas. This topic is further developed in [19], where the calculated and measured radiation patterns, as well as the resulting channel time dispersion characteristic, are given.

\subsection{Multibeam Lens Antennas}

The first example refers to an integrated multibeam lens antenna intended to produce $3 \mathrm{~dB}$ beamwidths on the order of $6^{\circ}$ at the $60-\mathrm{GHz}$ band, with high Gaussissity, gain scan loss lower than $1 \mathrm{~dB}$ over $\pm 20^{\circ}$ scan angle, and complying with a maximum diameter of $70 \mathrm{~mm}$ [59]. The lens was required to operate over $40 \%$ bandwidth, and thus, the challenge was to minimize internal reflections and to ensure a reasonably stable Gaussian beam shape across the scanned angular interval.

A double-shell configuration was adopted to allow imposing both a beam collimation condition and the Abbe sine condition to minimize aberration of the output beam. The selected lens materials were the MACOR/acrylic combination (permittivity 5.5/2.53), and the feed was the especially designed waveguide aperture described in Section 2 for $62.5-\mathrm{GHz}$ operation. Figure 13(a) shows the lens antenna prototype that was designed using ILASH [31]. The lens dimensions are $60 \mathrm{~mm}$ in diameter at the base and $37 \mathrm{~mm}$ in height; the outer shell material is transparent, and thus, it reveals the inner shell, which appears distorted due to refraction effects. The dedicated waveguide feeds from Section 2 were used forconvenience of the measurement setup, which is based on a waveguide.

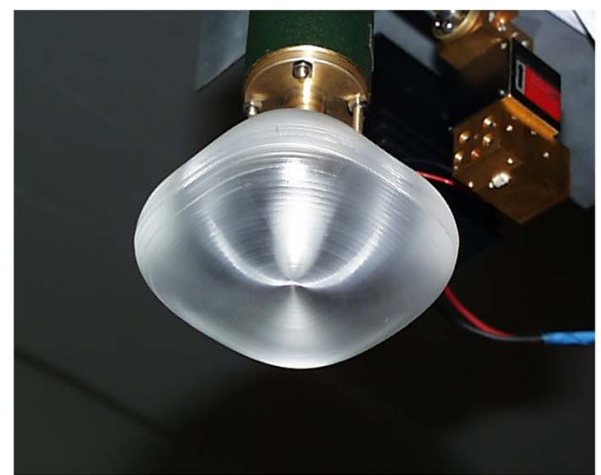

(a)

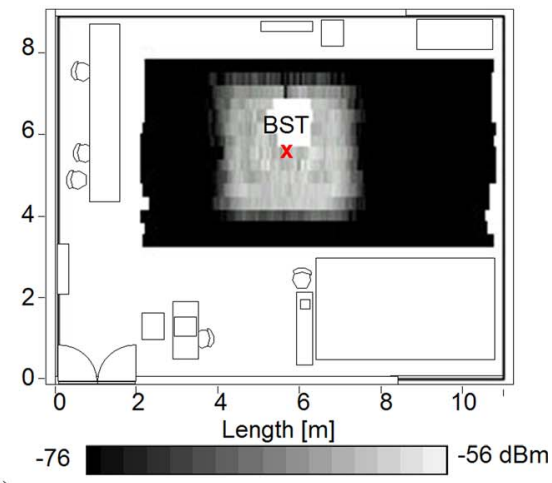

(b)

Figure 12. (a) Dielectric lens antenna for a square cell fed by a circular waveguide. (b) Received power distribution measured in the laboratory at $62.5 \mathrm{GHz}$. 


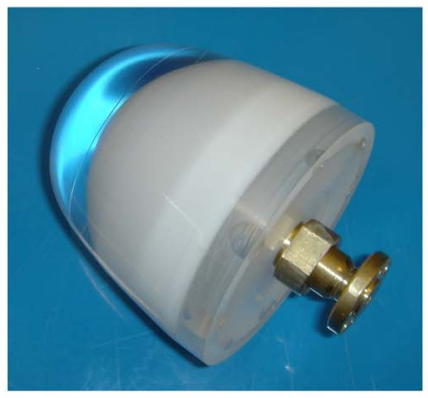

(a)

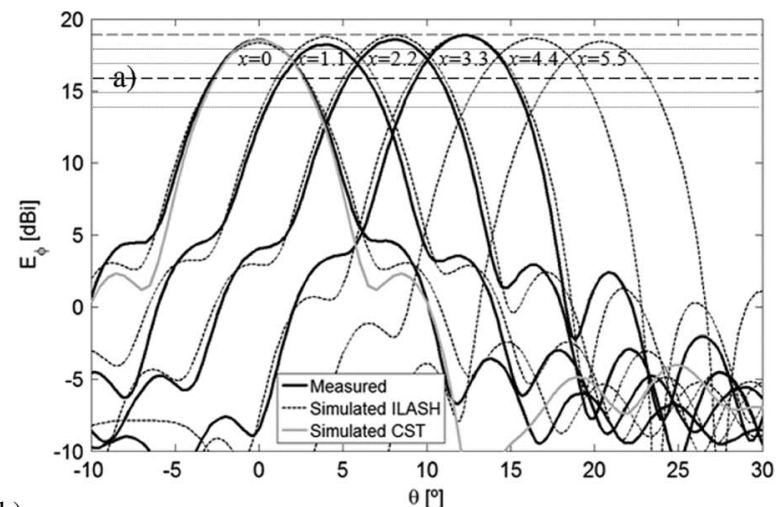

(b)

Figure 13. (a) Fabricated MACOR/acrylic lens prototype, showing the attached 60-GHz band waveguide feed. (b) Simulated and measured gain radiation patterns at $62.5 \mathrm{GHz}$, in the $\mathrm{H}$-plane, with feed at $0,1.1,2.2,3.3,4.4$, and $5.5 \mathrm{~mm}$ from the lens axis [31].

Figure 13(b) shows the measured and simulated radiation pattern results for different feed positions at $1.1-\mathrm{mm}$ steps along the lens base in the H-plane. This produces overlapping of consecutive beams near the $-1.5 \mathrm{~dB}$ level with respect to maximum. Beam overlap at $-3 \mathrm{~dB}$ occurs for wider separation between consecutive feeds, compatible with the used feed aperture size (not shown). The figure shows excellent agreement between ILASH predictions and measurements and confirms the effectiveness of the proposed double-shell lens concept regarding beam scan linearity, internal reflections, beam shape, and Gaussissity preservation with extremely low scan loss up to $20^{\circ}$ elevation. The achieved gain was on the order of $18-19 \mathrm{dBi}$, and the scan loss was better than $1 \mathrm{~dB}$ for $43-62 \mathrm{GHz}$. As a consequence of the GO-based lens design, the scan angle dependence on the feed off-axis position was practically independent of frequency, and the beam Gaussissity was better than $95 \%$.

The following example is intended to demonstrate the viability of the wideband XETS printed antenna with a simple integral mixer described in Section 2 as an integrated multibeam lens feed and to show the effectiveness of the IF signal retrieval setup. Despite the tightly packed assembly of the XETS elements at the lens base, the proposed feed still offers good isolation between adjacent elements both at RF and IF, providing very stable radiation pattern and linear polarization over the bandwidth.

The above objectives can be demonstrated using just a canonic single material lens, but the conclusions remain valid for more sophisticated lens design. A 68-mm-diameter MACOR elliptical lens was used in this case, as shown in Figure 14(a) [54]. The lens was designed to scan the angular interval from 0 to $\pm 18^{\circ}$ with beam overlap approximately at $-3 \mathrm{~dB}$ for $43 \mathrm{GHz}$ and at $-4.5 \mathrm{~dB}$ for $62.5 \mathrm{GHz}$. The overlap level at the higher frequencies could have been improved with other lens designs, but this type of optimization exceeded the established objective for this example.

The radiation pattern measurement configuration is shown in Figure 14(b). The Schottky diode integrated into the XETS receives both the $62.5-\mathrm{GHz}$ far-field TX signal (through the lens) and the 64-GHz LO signal (through the air from the back), producing the desired $1.5-\mathrm{GHz}$ IF signal. The LO assembly is fixed with respect to the lens, so that the LO signal amplitude illuminating the XETS does not change with lens rotation, whereas the IF signal amplitude changes only in response to lens radiation pattern at the TX frequency. (a)

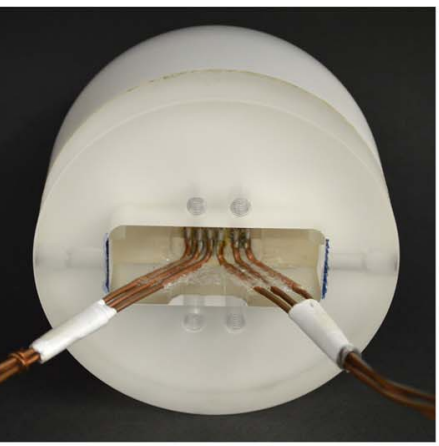

Rectangular waveguide $f_{\mathrm{LO}}=64 \mathrm{GHz}(\mathrm{LO})$

Rotates together with the lens

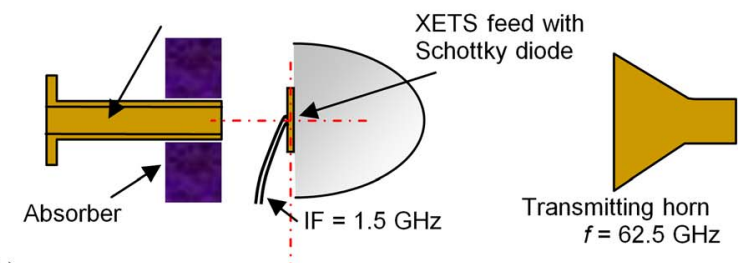

(b)

Figure 14. (a) Bottom view of a MACOR elliptical lens fed by a multi-XETS assembly with an integrated mixer. (b) Radiation pattern measurement setup at 62.5 GHz, using an integrated mixer at the base of the lens. 


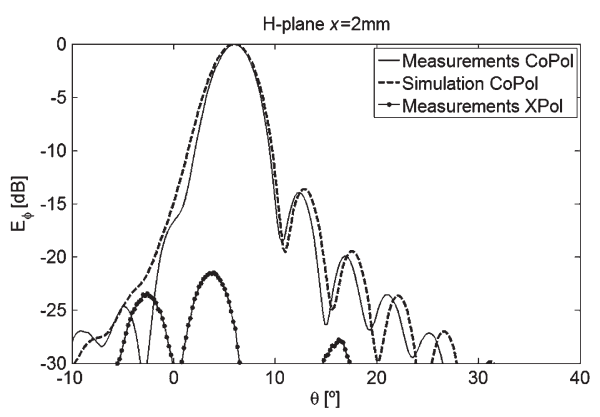

(a)

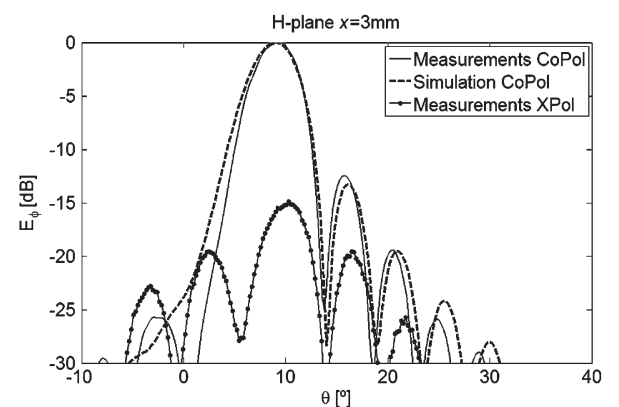

(b)

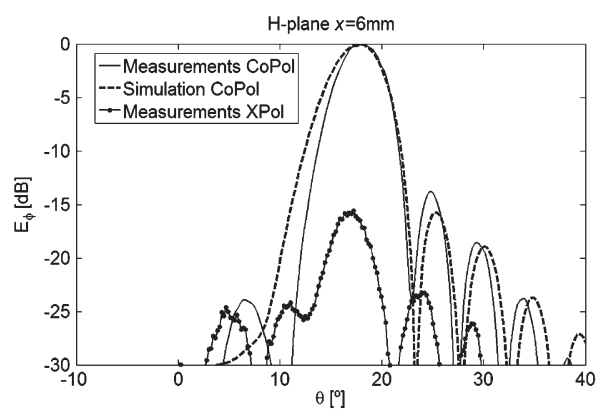

(c)

Figure 15. Comparison between simulated and measured radiation patterns at $62.5 \mathrm{GHz}$ in the $\mathrm{H}$-plane for a feed displacement of (a) $x=2 \mathrm{~mm}$, (b) $x=3 \mathrm{~mm}$, and (c) $x=6 \mathrm{~mm}$.

Examples of the H-plane measured and simulated radiation patterns are depicted in Figure 15 at $62.5 \mathrm{GHz}$ for three XETS elements $(x=2 \mathrm{~mm}, x=3 \mathrm{~mm}$, and $x=6 \mathrm{~mm})$. The curves are normalized to the respective maximum. The main lobe shape is quite stable, with the correct beam inclination despite the very close position of the XETS elements. Crosspolar level is below $-15 \mathrm{~dB}$, which is much better than what can be obtained, for instance, with the self-complementary log-periodic feed. This validates the expected feed performance. The only inconvenience of this feed is that, once glued at the lens base, it can no longer be reused.

\subsection{Broadband Lens as a Primary Feed for Reflectors}

A double-shell shaped dielectric lens with five integrated feeds at its base was designed to illuminate a $90^{\circ}$ offset reflector with slightly less than $2 \lambda$ separation between focal points, for $3 \mathrm{~dB}$ adjacent beam rollover. The lens output beams for each embedded feed produce a virtual focus located far below the lens and close to the corresponding reflector focal points [see Figure 16(a)]. The parabola rotates about an axis passing through the lens axis for mechanical beam scan in azimuth, whereas the lens provides multiple beam coverage in elevation. The challenge was to match the above requirements along with stable scanning characteristics, transmission efficiency, and aperture efficiency over the specified 1:3 bandwidth.

The shaped double-shell dielectric lens antenna was designed by imposing simultaneously a well-defined phase center located below the lens body and an aberration condition, as shown in Figure 16(b) [28]. The 20 $\lambda$ diameter lens prototype was made of MACOR and acrylic and was fed by the waveguide feed described in Section 2 with a special fixture to allow its precise positioning at different positions at the lens base. The lens performance in terms of radiation pattern and phase center measured at 43 and $62.5 \mathrm{GHz}$ agreed very well with the specifications [28]. The lens output beam Gaussissity was better than $94 \%$ over the band.

The performance of the lens-reflector assembly was simulated using the ILASH GO/PO module. The reflector radiation pattern at $62.5 \mathrm{GHz}$ is presented in Figure 17(a) showing well-defined beams with maximum $1.6 \mathrm{~dB}$ scan loss. Figure 17(b) shows for the central beam that the aperture efficiency is practically constant versus frequency, and in agreement with that property, the directivity in decibels increases almost linearly with frequency. (a)

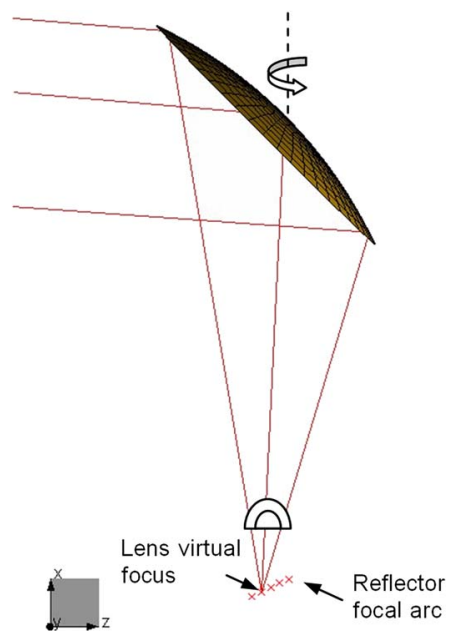

Figure 16. (a) Problem geometry. (b) Fabricated lens prototype with a MACOR inner shell and an acrylic outer shell.

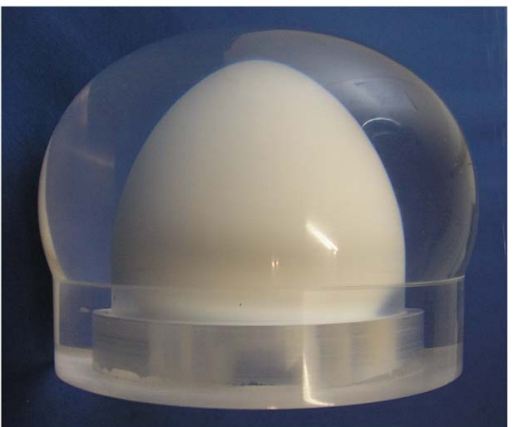

(b) 


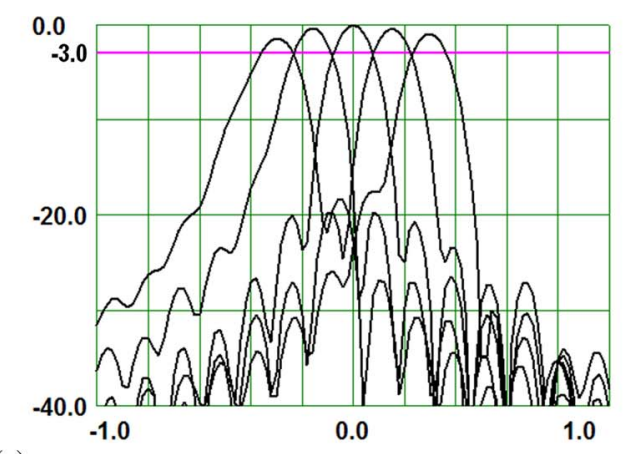

(a)

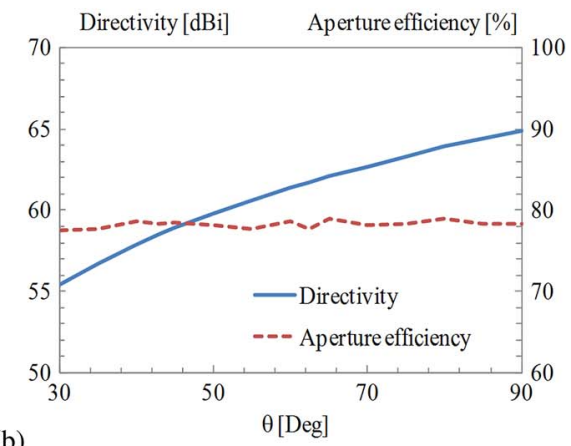

(b)

Figure $\subseteq$ Radiation pattern of the reflector when illuminated by the fabricated lens (a) Radiation pattern prototype with an inner shell of MACOR and an outer shell of acrylic. (b) Calculated directivity and aperture efficiency of the reflector for the central beam.

In a second example, the objective was to design a focal plane feed for a reflector such that the reflector radiation pattern has a frequency independent beamwidth over 1:3 bandwidth. It is known that the larger the reflector illuminated area is, the narrower is its radiation pattern. Hence, the reflector feed is required to have an increasing directivity for increasing frequencies. Simultaneously, it must exhibit a stable phase center across the whole band. The reflector is based on a $90^{\circ}$ offset configuration with focal length $F=3 \mathrm{~m}$ and projected aperture diameter $D=2.5 \mathrm{~m}$. A 70-mm-diameter double-shell collimated beam lens was designed using the MACOR/acrylic material combination, as shown in Figure 18(a) [27]. The performance of the isolated lens was experimentally characterized at 43 and $62.5 \mathrm{GHz}$ using the waveguide feeds described in Section 2 that provide a very similar radiation pattern at the two frequencies. The combined lens plus reflector performance was evaluated by simulation using ILASH. The reflector radiation pattern for different frequencies is shown in Figure 18b. Despite some variation of the lens phase center with frequency, its impact on the reflector directivity is not significant. It is on the order of $56 \mathrm{dBi}$ within the considered band, with a variation of $\pm 0.5 \mathrm{~dB}$. It is noted, however, that the success of the design is limited by the condition that the feed radiation pattern is constant over the band and that lens dissipation losses are very low, so that its frequency behavior is not manifested in the reflector gain characteristic.

\subsection{Mechanical Beam-Steering Lenses}

A new lens antenna configuration was proposed by the authors in [29] for low Earth orbit satellite data link communications at $26 \mathrm{GHz}$. The lens was required to produce a sector beam with shaping in elevation and to enable a simple mechanical azimuth scanning. The target gain versus elevation angle $\theta$ is given approximately by $\sec (k \theta)$. With an appropriate choice of the $k$ shaping parameter, this elevation radiation pattern compensates path-loss attenuation taking into account the Earth's curvature.

The designed shaped lens is axial symmetric, but a spherical air cavity is excavated through its base at a calculated offset distance with respect to the lens symmetry axis [see Figure 19 (a)]. The feed aperture is at the center of this spherical air cavity. The asymmetry introduced by the feed position produces an adequate directive shaped beam [see Figure 19(b)]. This is an extremely simple solution where the feed is fixed and the lens rotates about the feed axis to provide the required beam scanning.

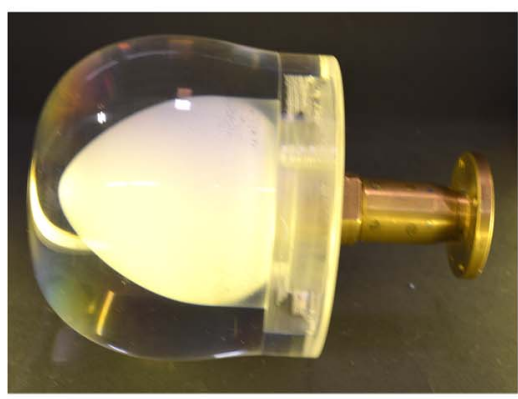

(a)

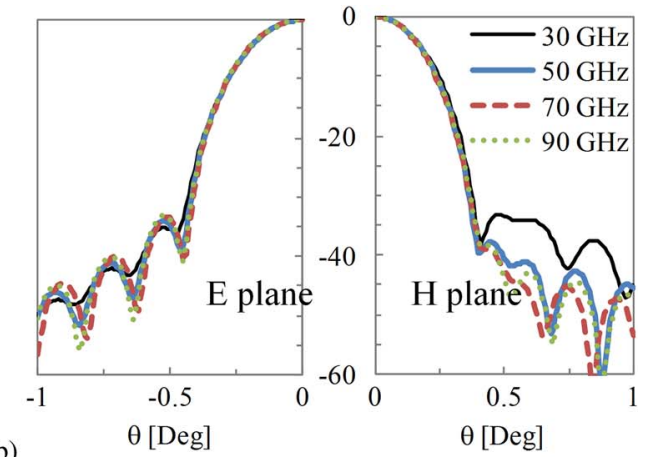

(b)

Figure 18. (a) Fabricated MACOR/acrylic lens prototype, showing the attached 60-GHz band waveguide feed. (b) Simulated radiation patterns of the reflector fed by the lens. 

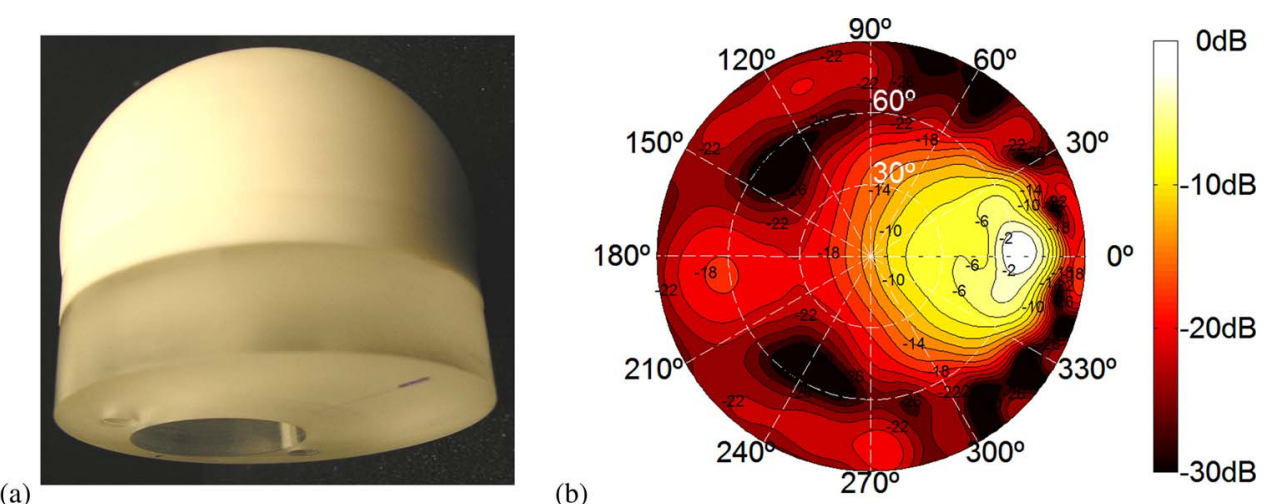

Figure 19. (a) MACOR axial-symmetric 50-mm-diameter shaped lens. (b) Radiation performance (in polar coordinates) of the lens computed with the GO/PO method.

The feed is not in physical contact with the lens, thus avoiding the need for fault-prone rotary joints in long continuous usage. The assembly is very compact and lightweight (lens diameter is less than five wavelengths, and height is less than three wavelengths) complying with mass and volume restrictions for satellites.

The same concept of a moving lens with fixed feed was extended for azimuth plus elevation mechanical scanning in a different application context. Wireless indoor transmission of an uncompressed high-definition (HD) video signal between a video device such as a camcorder and an HD TV display may soon become a requirement for mass market home applications. The WirelessHD standard [70] proposes to use the unlicensed spectrum from 57 to $66 \mathrm{GHz}$ for such applications. For mass consumer applications, the radio link must use inexpensive low-power sources, and hence, high gain antennas $(>20 \mathrm{dBi})$ are required to favor the link budget. The narrow beam needs to be steered to allow for the user mobility. A new configuration of a mechanical steerable beam antenna has been developed at IT where a dielectric lens pivots in front of a single stationary moderate gain feed, as shown in Figure 20(a) [30]. Again, the feed is not in physical contact with the lens. As discussed previously, this eliminates the need for rotary joints and the associated drawbacks. The designed lens is made of polyethylene and is such that both the input and output lens surfaces are shaped to accommodate a beam collimation condition and a scan angle maximization condition. The lens output beam is aligned with the lens axis for all lens orientations within the operation cone. Thus, pivoting the lens around two of its main axis allows steering the beam in elevation and azimuth. The important difference with respect to conventional scanning lenses is that, for all inclinations of the lens, the focus is always exactly coincident with the aperture phase center. In this way, aberrations are strongly reduced, allowing wider scan angles than conventional solutions. The fabricated lens assembly demonstrated a $-45^{\circ}$ to $+45^{\circ}$ elevation scan capability over full azimuth, with $21 \mathrm{~dB}$ gain and better than $-1.1 \mathrm{~dB}$ gain scan loss [see Figure 20(b)]. The radiation efficiency was always above $95 \%$. A simple system demonstrator has been recently assembled to prove the feasibility of the antenna concept, of the tracking mechanism, and its algorithms [71].

\subsection{Near-Field Lens Operated in Backscattering Mode}

Next, we report some of our findings in the area of near-field lensing. In this context, the main challenge is to produce a very localized beam spot that can be used to probe (a)

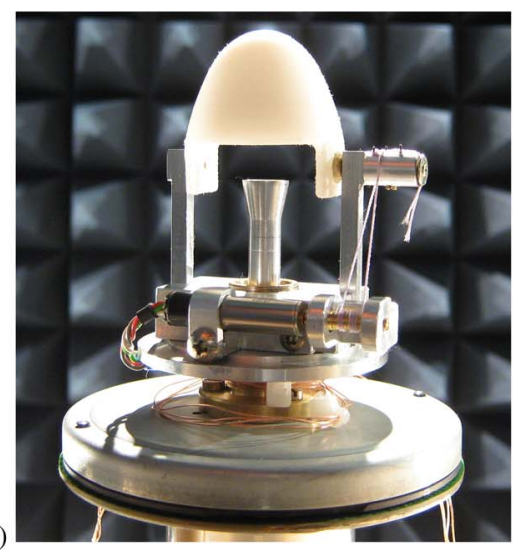

(b)

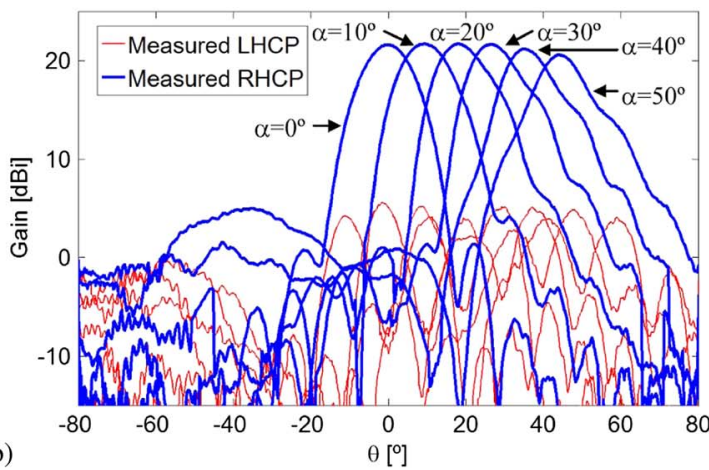

Figure 20. (a) Photograph of the manufactured polyethylene lens plus horn feed. (b) Measured radiation patterns of the lens antenna for several lens tilt angles. 


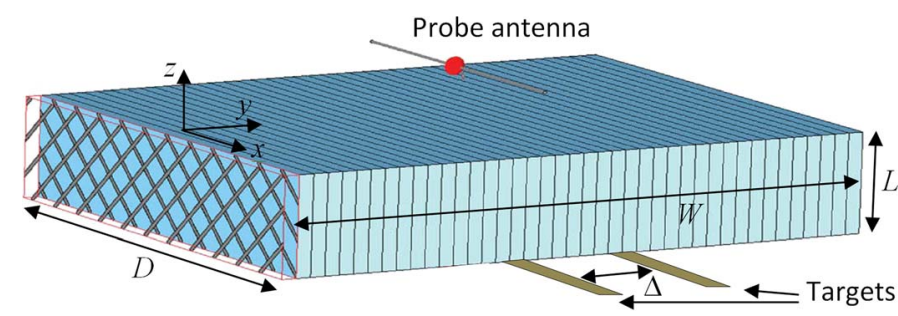

Figure 21. Geometry of the crossed-wire slab showing also the probe antenna and two metal strip targets.

subwavelength details of an object or to detect very closely spaced objects. This can be interesting for many applications, which include subwavelength imaging, detection of buried targets, microwave heating, etc. To manipulate and resonantly interact with the near field, one can rely on metamaterials. Near-field lenses made of metamaterials have been extensively studied in recent years. Some examples include wire media lenses [72]-[74], high-pass spatial filtering lenses [75] based on magnetoinductive waves [76], lenses made of left-handed materials and negative permittivity materials [77]-[80], hyperbolic media lenses [81], [82], and structures relying on radiationless electromagnetic interference [83]-[86].

We have demonstrated in [84] that materials with an extremely large and positive index of refraction may provide an interesting solution to collimate the near field, preventing a lateral spreading of the beam. Such materials may be implemented in practice based on the crossed-wire medium metamaterial. We have shown that a dense metallic mesh of nonconnected crossed wires can support guided modes associated with extremely short guided wavelengths, and these guided modes may be resonantly coupled to the near field, somewhat analogous to surface plasmon polaritons [85], [86]. Because of these properties, a thin slab of our metamaterial "superlens" may enhance subwavelength features inaccessible with conventional systems [84].

As an example, we report in Figure 21 a prototype of a crossed-wire metamaterial lens fabricated at IT. It is formed by a stack of 308 rectangular printed circuit boards of ROGERS duroid 5880 with dielectric constant $\varepsilon_{r}=2.2$, loss tangent $\tan \delta=0.0009$, thickness $0.79 \mathrm{~mm}$, and dimensions $D=182 \mathrm{~mm}$ and $L=11.7 \mathrm{~mm}$. Each board is printed with an array of parallel metallic strips tilted sequentially by $-45^{\circ}$ and $+45^{\circ}$ with respect to the $z$-direction. The width of the printed strips is $w_{s}=0.247 \mathrm{~mm}$, and the repetition step is $\alpha=1.58 \mathrm{~mm}$. Here, we describe the operation of the lens in backscattering mode. In this context, a dipole is used as probe antenna in front of the lens, and two 2.5-mm-wide metal strips are used as targets behind the lens, all aligned with the $x$-coordinate as required by the polarization-selective metamaterial lens. The lens assembling process is shown in Figure 22(a), and the details of the measurement setup are shown in Figure 22(b) and Figure 22(c).

As demonstrated in our previous works [84], the crossedwire lens enables collimating the near field into a subwavelength spot. This happens both with the incident field at the target and with the backscattered field at the probe antenna position. These near-field interactions affect the input impedance of the probe antenna. Thus, the target position can be determined simply by detecting changes on the return loss of the probe antenna as it scans the $x$-direction. For the tests, the target metal strips were positioned at $z=-3 L / 2$, successively with spacing $\Delta=7.0,4.7$, and $3.5 \mathrm{~cm}$ (first, second, and third rows of Figure 23, respectively). These correspond to $\Delta=0.3 \lambda_{0}$, $0.2 \lambda_{0}$, and $0.15 \lambda_{0}$ at $1.28 \mathrm{GHz}$, the resonance frequency of the dipole antenna in free space.

Figure 23 depicts the perturbation of the input reflection coefficient $\left|s_{11}\right|_{d B}$ measured at the dipole probe port versus its $y / \lambda_{0}$ position at a constant height $z=0.5 \mathrm{~L}$ (blue, solid line). The red dash-dotted line corresponds to the E-field calculated from a theoretical model under the same conditions. The green dashed line represents the measured perturbation of $\left|s_{11}\right|_{d B}$ after removing the lens. The vertical dashed lines mark the targets' position.

It is shown in Figure 23 that the system clearly discriminates the targets for $\Delta=0.3 \lambda_{0}$ and $0.2 \lambda_{0}$, even for $\Delta=0.15 \lambda_{0}$, and discrimination is almost possible at $1.20 \mathrm{GHz}$. The targets cannot be resolved when the lens is removed. It is worth mentioning that the lens resolving power may be improved by increasing the number of wires per unit of volume.

Unlike other works where the superlensing has been demonstrated over narrow bandwidth based on a negative refractive index, this lens exhibits superlensing at microwaves based on the (extremely high) positive effective refractive

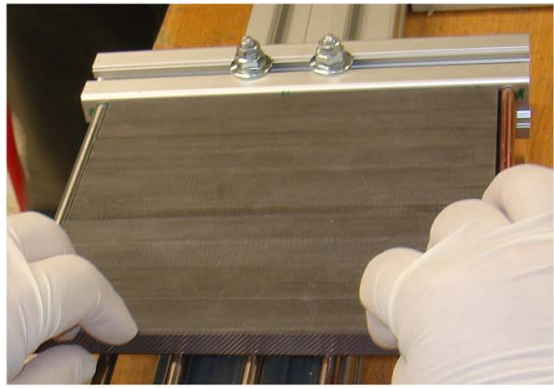

(a)

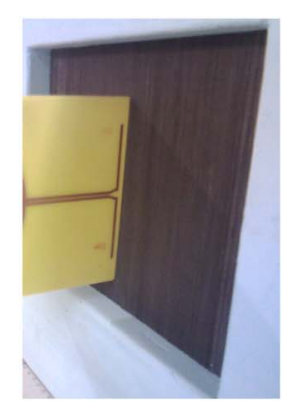

(b)

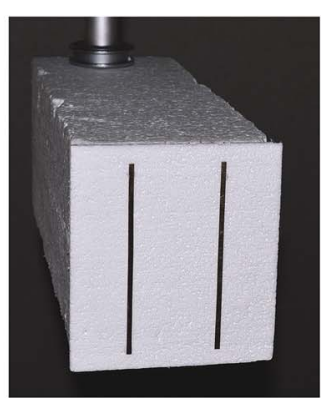

(c)

Figure 22. (a) Fabrication of the multilayer crossed-wire lens. (b) Detail of the probing antenna. (c) Metal strips used as backscatter targets. 

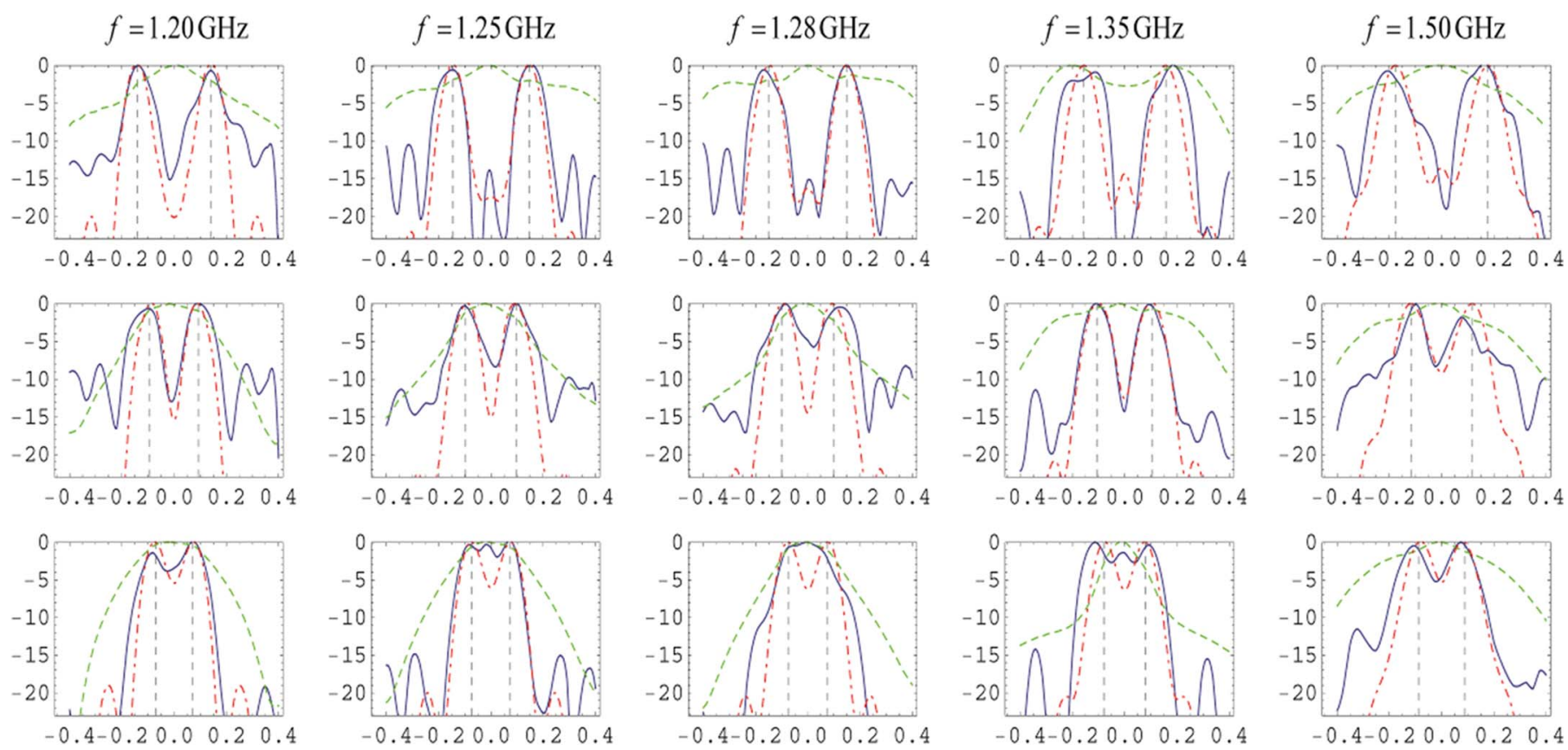

Figure 23. Measured perturbation of the $\left|s_{11}\right|_{d B}$ at the dipole probe port versus its $y / \lambda_{0}$ position, at five frequencies (columns) and three target $\Delta$ spacings (rows). Solid (blue) lines: measured results with the lens; dashed (green) lines: measured results without the lens; the dot-dashed (red) lines: calculated with a theoretical model (for the lens setup). The dashed (gray) vertical lines represent the position of the two targets.

index metamaterial. It has about $25 \%$ bandwidth due to the nonresonant nature of the metamaterial. Here, we described its operation in backscattering mode having in mind further studies for imaging applications.

\section{Conclusion}

Since 1992, IT has been actively and continually involved in the development of dielectric lens antennas in the framework of several national and international research projects. Pioneering work was done in the 1990s on highly shaped integrated lens antennas for constant flux wireless applications at $60 \mathrm{GHz}$. Later on, this effort was extended to double-shell shaped integrated lenses to meet more demanding antenna performance requirements. The work was also extended to nonuniform index lenses, aiming at planar configurations for easier integration with planar monolithic feeding circuits. Analytical formulations based on GO were derived to provide a first guess solution for numerous cases of interest, with computation times on the order of seconds. Some of these solutions were good enough for the final application without the need for additional design refinement. A parallel work has been carried out to design dedicated high-performance feeds for integrated lenses. Recent work that is still active is on metamaterial near-field lenses.

Based on the accumulated experience, a powerful shaped lens design and optimization tool with a user-friendly interface-ILASH - was developed at IT that includes the mentioned closed-form formulations, as well as efficient GA optimization tools for lens synthesis. ILASH includes also standard methods for lens design diagnose and performance evaluation not only as a stand-alone antenna but also in combination with a parabolic reflector.

A couple of interesting and innovative fully functional lens examples were discussed in this work to illustrate the research path on lenses at IT over the years and simultaneously to show the design flexibility of lenses for accommodating very different specifications in very different applications.

\section{Acknowledgments}

The authors acknowledge the collaboration from V. Fred and C. Brito for prototype construction, as well as A. Almeida and J. Silva for prototype measurements. The authors would also like to thank M. van der Vorst for discussions on some aspects of the lens design, R. Sauleau for providing access to his laboratory for complementary measurements on a selected prototype, and C. R. Medeiros for support with the metamaterial lens measurement setup, as well as numerous students that actively contributed to this work in the framework of their theses. The work was supported by several projects, including mmSatCom PTDC/EEI-TEL/0805/2012 and NEWFOCUS ESF RNP.

\section{References}

[1] Instituto de Telecomunicações. [Online]. Available: www.it.pt.

[2] F. Friedlander, "A dielectric-lens aerial for wide-angle beam scanning," J. Inst. Elect. Eng.-III A, Radiolocation, vol. 93, no. 4, pp. 658-662, 1946.

[3] J. Brown, Microwave Lenses. London, U.K.: Wiley, 1953.

[4] S. Cornbleet, Microwave and Geometrical Optics. San Diegpo, CA, USA: Academic Press, 1994. 
[5] J. Lee, "Lens antennas," Chapter 16 in Antenna Handbook-Theory, Applications and Design, Y. T. Lo and S. W. Lee, Eds., New York, NY, USA: Van Nostrand, 1988.

[6] C. Sletten, Reflector and Lens Antenna. Norwood, MA, USA: Artech House, 1988.

[7] D. B. Rutledge and M. S. Muha, "Imaging antenna arrays," IEEE Trans. Antennas Propag., vol. AP-30, no. 4, pp. 535-540, Jul. 1982.

[8] G. Rebeiz, "Millimeter-wave and terahertz integrated circuit antennas," Proc. IEEE, vol. 80, no. 11, pp. 1748-1770, Nov. 1992.

[9] L. Fernandes, "Developing a system concept and technologies for mobile broadband communications," IEEE Pers. Commun. Mag., vol. 2, no. 1, pp. 54-59, Feb. 1995.

[10] C. Fernandes, P. Francês, and A. Barbosa, "Shaped coverage of elongated cells at millimetre waves using a dielectric lens antenna," in Proc. $E M C$, Bologna, Italy, Sep. 1995, vol. 1, pp. 66-70.

[11] D. Lemaire, C. Fernandes, P. Sobieski, and A. Barbosa, "A method to overcome the limitations of G.O. in the design of axis-symmetrical lenses," Int. J. Infrared Millim., vol. 17, no. 8, pp. 1377-1390, Aug. 1996.

[12] C. Fernandes, "Shaped dielectric lenses for wireless millimeterwave communications," IEEE Antennas Propag. Mag., vol. 41, no. 5, pp. 141-150, Oct. 1999.

[13] D. Filipovic, S. Gearheart, and G. Rebeiz, "Double-slot antennas on extended hemispherical and elliptical silicon dielectric lenses," IEEE Trans. Microw. Theory Tech., vol. 41, no. 10, 1738-1749, Oct. 1993.

[14] C. Salema, C. Fernandes, and R. Jha, Solid Dielectric Horns, Boston, MA, USA: Artech House, ch. 7, 1998.

[15] C. Fernandes, "Shaped-beam antennas," in Handbook of Antennas in Wireless Communications, L. Godara, Ed., New York, NY, USA: CRC Press, ch. 152001.

[16] J. Zubrzycki, "Mobile broadband system-A report on the work of race project 2067," British Broadcasting Corporation RD 1996/12, 1996. [Online]. Available: http://archive.org/details/bbc-rd-reports-1996-12.

[17] A. Plattner, B. Byzery, C. Femandes, and T. Karttaavi, "A compact portable $40 \mathrm{GHz}$ transceiver for the mobile broadband system," in Proc. ACTS Mobile Commun. Summit, Rhodes, Greece, Jun. 1998, pp. 843-848.

[18] M. Prögler, "SAMBA: A mobile broadband enabler," in Proc. Eur. Microw. Conf., Munich, Germany, Oct. 1999, pp. 87-90.

[19] C. Fernandes and L. Anunciada, "Constant flux illumination of square cells for mm-wave wireless communications," IEEE Trans. Microw. Theory Tech., vol. 49, no. 11, pp. 2137-2141, Nov. 2001.

[20] C. Fernandes and J. Fernandes, "Performance of lens antennas in wireless indoor millimeter-wave applications," IEEE Trans. Microw. Theory Tech., vol. 47, no. 6, pp. 732-737, Jun. 1999.

[21] C. Fernandes, "Design of shaped lenses for non-symmetric cells in MBS," in Proc. IEEE Int. Symp. Antennas Propag., Orlando, FL, USA, Jul. 1999, pp. 2440-2443.

[22] M. Silveirinha and C. Fernandes, "Shaped double-shell dielectric lenses for wireless millimetre wave communications," in Proc. IEEE Int. Symp. Antennas Propag., Salt Lake City, UT, USA, 2000, pp. 1674-1677.

[23] P. Siegel, "THz instruments for space," IEEE Trans. Microw. Theory Tech., vol. 55, no. 11, pp. 2957-2965, Nov. 2007.

[24] A. Semenov, G. Gol'tsman, and R. Sobolevski, "Hot-electron effect in superconductors and its application for radiation sensors," Supercond. Sci. Technol., vol. 15, no. 4, pp. R1-R16, Apr. 2002.

[25] L. Liu et al., "Development of integrated terahertz broadband detectors utilizing superconducting hot-electron bolometers," IEEE Trans. Appl. Supercond., vol. 19, no. 3, pp. 282-286, Jun. 2009.

[26] A. Semenov et al., "Terahertz performance of integrated lens antennas with a hot-electron bolometer," IEEE Trans. Microw. Theory Tech., vol. 55, no. 2, pp. 239-247, Feb. 2007.

[27] E. Lima, J. Costa, and C. Fernandes, "Broadband reflector fed by integrated lens antenna with frequency constant directivity," in Proc. IEEE Int. Symp. Antennas Propag., Toronto, OM, Canada, Jul. 2010, pp. $1-4$.

[28] C. Fernandes, E. Lima, and J. Costa, "Broadband integrated lens for illuminating reflector antenna with constant aperture efficiency," IEEE Trans. Antennas Propag., vol. 52, no. 12, pp. 3805-3813, Dec. 2010.

[29] J. Costa et al., "Compact Ka-band lens antennas for LEO satellites," IEEE Trans. Antennas Propag., vol. 56, no. 6, pp. 1251-1268, May 2008.

[30] J. Costa, E. Lima, and C. Fernandes, "Compact beam-steerable lens antenna for $60 \mathrm{GHz}$ wireless communications," IEEE Trans. Antennas Propag., vol. 57, no. 10, pp. 2926-2933, Oct. 2009.

[31] E. Lima, J. Costa, M. Silveirinha, and C. Fernandes, "ILASHSoftware tool for the design of integrated lens antennas," in Proc. IEEE Int. Symp. Antennas Propag., San Diego, CA, USA, Jul. 2008, pp. $1-4$.

[32] M. van der Vorst, P. de Maagt, and M. Herben, "Matching layers for integrated lens antennas," in Proc. JINA, Nice, France, 1996, pp. 511-515.
[33] A. Neto, S. Maci, and P. de Maagt, "Reflections inside an elliptical dielectric lens antenna," Proc. Inst. Elect. Eng.-Microw. Antennas Propag., vol. 145 , no. 3, pp. 243-247, Jun. 1998.

[34] A. Neto, D. Pasqualini, A. Toccafondi, and S. Maci, "Mutual coupling between slots printed at the back of elliptical dielectric lenses," IEEE Trans. Antennas Propag., vol. 47, no. 10, pp. 1504-1507, Oct. 1999.

[35] M. van der Vorst, P. de Maagt, and M. Herben, "Effect of internal reflections on the radiation properties and input admittance of integrated lens antennas," IEEE Trans. Microw. Theory Tech., vol. 47, no. 9, pp. 1696-1704, Sep. 1999.

[36] J. Bray and L. Roy, "Analysis of electrically small slot-fed substrate lens antennas using the physical optics hybrid method," Electromagnetics, vol. 20, no. 3, pp. 243-265, May 2000.

[37] M. van der Vorst et al., "Effect of internal reflections on the radiation properties and input impedance of integrated lens antennas - Comparison between theory and measurements," IEEE Trans. Microw. Theory Tech., vol. 49 , no. 6 , pp. 27-32, Sep. 2001.

[38] D. Pasqualini and S. Maci, "High-frequency analysis of integrated dielectric lens antennas," IEEE Trans. Antennas Propag., vol. 52, no. 3, pp. 840-847, Mar. 2004.

[39] A. P. Pavacic, D. L. del Rio, J. R. Mosig, and G. V. Eleftheriades, "Three-dimensional ray-tracing to model internal reflections in offaxis lens antennas," IEEE Trans. Antennas Propag., vol. AP-54, no. 2, pp. 604-612, Jul. 2009.

[40] N. T. Nguyen, R. Sauleau, and C. J. M. Perez, "Very broadband extended hemispherical lenses: Role of matching layers for bandwidth enlargement," IEEE Trans. Antennas Propag., vol. AP-57, no. 7, pp. 1907-1913, Jul. 2009.

[41] A. Neto, "UWB, non dispersive radiation from the planarly fed leaky lens antenna-Part I: Theory and design," IEEE Trans. Antennas Propag., vol. AP-58, no. 7, pp. 2238-2247, Jul. 2010.

[42] A. Neto, S. Monni, and F. Nennie, "UWB, non dispersive radiation from the planarly fed leaky lens antenna-Part II: Demonstrators and measurements," IEEE Trans. Antennas Propag., vol. AP-58, no. 7, pp. 2248-2258, Jul. 2010.

[43] N. Llombart, G. Chattopadhyay, A. Skalare, and I. Mehdi, "Novel terahertz antenna based on a silicon lens fed by a leaky wave enhanced waveguide," IEEE Trans. Antennas Propag., vol. 59, no. 6, pp. 2160-2168, Jun. 2011.

[44] B. Barès, R. Sauleau, L. Le Coq, and K. Mahdjoubi, "A new accurate design method for millimeter-wave homogeneous dielectric substrate lens antennas of arbitrary shape," IEEE Trans. Antennas Propag., vol. 53, no. 3, pp. 1069-1082, Mar. 2005.

[45] G. Godi, R. Sauleau, R. L. Le Coq, and D. Thouroude, "Design and optimization of three-dimensional integrated lens antennas with genetic algorithm," IEEE Trans. Antennas Propag., vol. 55, no. 3, pp. 770-775, Mar. 2007.

[46] N. T. Nguyen et al., "Design and characterization of $60-\mathrm{GHz}$ integrated lens antennas fabricated through ceramic stereolithography," IEEE Trans. Antennas Propag., vol. 58, no. 8, pp. 2757-2762, Aug. 2010.

[47] M. Silveirinha and C. Fernandes, "Cylindrical non-homogeneous anisotropic lenses using artificial media," in Proc. IEEE Int. Symp. Antennas Propag., Boston, MA, USA, Jul. 2001, vol. 1, pp. 312-315.

[48] M. Silveirinha and C. Fernandes, "Design of a non-homogeneous wire media lens using genetic algorithms," in Proc. IEEE Int. Symp. Antennas Propag., San Antonio, TX, USA, Jun. 2002, vol. 1, pp. 730-733.

[49] M. G. Silveirinha, "Electromagnetic waves in artificial media with application to lens antennas," Ph.D. Dissertation, Inst. Superior Técnico, Tech. Univ. Lisbon, Lisbon Portugal, Apr. 2003.

[50] M. Silveirinha and C. Fernandes, "Homogenization of metamaterial surfaces and slabs: The crossed wire mesh canonical problem," IEEE Trans. Antennas Propag., vol. 53, no. 1, pp. 59-69, Jan. 2005.

[51] J. Costa, M. Silveirinha, and C. Fernandes, "Design and analysis of double-shell axial-symmetric integrated lens antennas for space applications," in Proc. ESA Antenna Workshop Space Antenna Syst. Technol., Noordwijk, The Netherlands, Jun. 2005, pp. 865-872, vol. 2.

[52] C. Fernandes, E. Lima, and J. Costa, "Tapered waveguide feed for integrated dielectric lens antenna performance tests," in Proc. EUROCON CONFTELE, Lisbon, Portugal, Apr. 2011.

[53] J. Costa and C. Fernandes, "Broadband slot feed for integrated lens antennas," IEEE Antennas Wireless Propag. Lett., vol. 6, pp. 396-400, 2007.

[54] J. Costa and C. Fernandes, "Integrated imaging lens antenna with broadband feeds," in Proc. EUCAP, Edinburgh, U.K., Nov. 2007, pp. 1-6.

[55] M. Silveirinha, C. A. Fernandes, and J. R. Costa, "A Graphical Aid for the Complex Permittivity Measurement at Microwave and Millimeter Wavelengths," IEEE Microwave Wireless and Components Letters, vol. 24, no. 6, pp. 421-423, May 2014. 
[56] M. Afsar, X. Li, and H. Chi, "An automated $60 \mathrm{GHz}$ open resonator system for precision dielectric measurement," IEEE Trans. Microw. Theory Tech., vol. 38, no. 12, pp. 1845-1853, Dec. 1990.

[57] C. Fernandes and J. Costa, "Permittivity measurement and anisotropy evaluation of dielectric materials at millimeter-waves," in Proc. IMEKO World Congr., Lisboa, Portugal, pp. 673-677, Sep. 2009.

[58] Rogers Corporation. [Online]. Available: http://www.rogerscorp.com.

[59] J. Costa, M. Silveirinha, and C. Fernandes, "Evaluation of a doubleshell integrated scanning lens antenna," IEEE Antennas Wireless Propag. Lett., vol. 7, pp. 781-784, 2008.

[60] E. Lima, J. Costa, and C. Fernandes, "Design of double material integrated scanning lens antennas," in Proc. IEEE Int. Symp. Antennas Propag., Charleston, SC, USA, Jun. 2009, pp. 1-4.

[61] C. Fernandes, J. Costa, and M. Vorst, "Design of a shaped doubleshell lens feed for a quasi-optical reflector system," in Proc. IEEE Int. Symp. Antennas Propag., Honolulu, United States, Jun. 2007.

[62] G. Godi, R. Sauleau, and D. Thouroude, "Performance of reduced size substrate lens antennas for millimeter-wave communications," IEEE Trans. Antennas Propag., vol. 53, no. 4, pp. 1278-1286, Apr. 2005.

[63] J. Costa and C. Fernandes, "Analysis of small integrated lens antennas using multiple point source physical optics discretization of the feed aperture field," in Proc. JINA, Nice, France, pp. 466-467, Nov. 2004.

[64] Computer Simulation Technology (CST), http://www.cst.com/.

[65] M. G. Silveirinha, C. Medeiros, C. Fernandes, and J. Costa, "Experimental verification of broadband superlensing using a metamaterial with an extreme index of refraction," Phys. Rev. B, Condens. Matter, vol. 81, no. 033101 , pp 1-4, Jan. 2010.

[66] M. G. Silveirinha, C. Medeiros, C. Fernandes, and J. Costa, "Resolving subwavelength objects with a crossed wire mesh superlens operated in backscattering mode," New J. Phys., vol. 13, no. 053004, pp. 1-14, May 2011.

[67] M. Kline, I. W. Kay, "Electromagnetic theory and geometrical optics," in Series Texts and Monographs on Pure and Applied Mathematics, vol. XII, Hoboken, NJ, USA: Wiley, 1965.

[68] M. Kharadly and W. Jackson, "The properties of artificial dielectrics comprising arrays of conducting elements," J. Inst. Elect. Eng., vol. 1953, no. 7, pp. 199-212, Jul. 1953.

[69] M. G. Silveirinha and C. Fernandes, "Transverse average field approach for the characterization of thin metamaterial slabs," Phys. Rev. E, vol. 75, no. 036613, pp 1-17, Mar. 2007.

[70] WirelessHD Specification Version 1.1 Overview, May 2010.

[71] J. Costa et al., "Development of an indoor wireless personal area network based on mechanically steered millimeter-wave lens antenna," in Proc. IEEE I2MTC, Austin, TX, USA, May 2010, vol. 1, pp. 1202-1206.

[72] P. A. Belov, Y. Hao, and S. Sudhakaran, "Subwavelength microwave imaging using an array of parallel conducting wires as a lens," Phys. Rev. B, Condens. Matter, vol. 73, no. 3, pp. 033108-033111, Jan. 2006.

[73] G. Shvets, S. Trendafilov, J. B. Pendry, and A. Sarychev, "Guiding, focusing, and sensing on the subwavelength scale using metallic wire arrays," Phys. Rev. Lett., vol. 99, no. 5, pp. 053903-053906, Aug. 2007.

[74] M. G. Silveirinha, P. A. Belov, and C. R. Simovski, "Subwavelength imaging at infrared frequencies using an array of metallic nanorods," Phys. Rev. B, Condens. Matter, vol. 75, no. 3, pp. 035108-035119, Jan. 2007.

[75] G. Fedorov et al., "Subwavelength imaging: Resolution enhancement using metal wire gratings," Phys. Rev. B, Condens. Matter, vol. 73, no. 3, pp. 035409-035415, Jan. 2006.

[76] M. J. Freire, R. Marques, and L. Jelinek, "Experimental demonstration of a $\mu=-1$ metamaterial lens for magnetic resonance imaging," Appl. Phys. Lett., vol. 93, no. 231 108, Dec. 2008.

[77] J. B. Pendry, "Negative refraction makes a perfect lens," Phys. Rev. Lett., vol. 85, no. 18, pp. 3966-3969, Oct. 2000.

[78] A. Grbic and G. V. Eleftheriades, "Overcoming the diffraction limit with a planar left-handed transmission-line lens," Phys. Rev. Lett., vol. 92 , no. 11 , pp. 117403 , Mar. 2004.

[79] N. Fang, H. Lee, C. Sun, and X. Zhang, "Sub-diffraction-limited optical imaging with a silver superlens," Science, vol. 308 , no. 5721, pp. 534-537, Apr. 2005

[80] T. Taubner, D. Korobkin, Y. Urzhumov, G. Shvets, and R. Hillenbrand, "Near-field microscopy through a $\mathrm{SiC}$ superlens," Science, vol. 313, no. 5793 , pp. 1595 , Sep. 2006.
[81] I. I. Smolyaninov, Y.-J. Hung, and C. C. Davis, "Magnifying superlens in the visible frequency range," Science, vol. 315 , no. 5819 , pp. 1699-1701, Mar. 2007.

[82] Z. Liu, H. Lee, Y. Xiong, C. Sun, and X. Zhang, "Far-field optical hyperlens magnifying sub-diffraction-limited objects," Science, vol. 315, no. 5819, p. 1686, Mar. 2007.

[83] A. Grbic, L. Jiang and R. Merlin, "Near-field plates: Subdiffraction focusing with patterned surfaces," Science, vol. 320, no. 5874, pp. 511-513, Apr. 2008.

[84] M. G. Silveirinha, C. Fernandes, and J. Costa, "Superlens made of a metamaterial with extreme effective parameters," Phys. Rev. B, Condens. Matter, vol. 78, no. 19, pp. 195121-195126, Nov. 2008.

[85] M. G. Silveirinha, C. A. Fernandes, "Nonresonant structured material with extreme effective parameters," Phys. Rev. B, Condens. Matter, vol. 78, no. 3, pp. 033108-033111, Jul. 2008.

[86] M. G. Silveirinha, C. A. Fernandes, J. R. Costa, C. R. Medeiros, "Experimental demonstration of a structured material with extreme effective parameters at microwaves," Appl. Phys. Lett., vol. 93, no. 17, pp. 174103-174105, Oct. 2008.

\section{Introducing the Feature Article Authors}

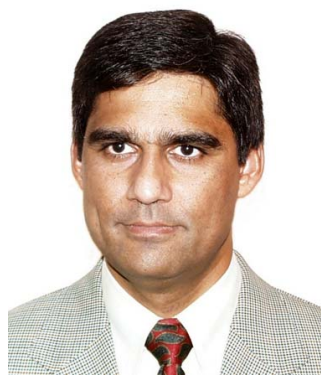

Carlos A. Fernandes received the Licenciado, M.Sc., and Ph.D. degrees from the Technical University of Lisbon, Lisbon, Portugal, in 1980, 1985, and 1990, respectively, all in electrical and computer engineering.

He joined the Instituto Superior Técnico, Technical University of Lisbon, in 1980, where he is currently a Full Professor at the Department of Electrical and Computer Engineering in the areas of microwaves, radio wave propagation, and antennas. He is a member of the Board of Directors of the research institute Instituto de Telecomunicaçoes and the Coordinator of its Wireless Communications Scientific Area. He has been the leader of antenna activities in national and European projects, such as RACE 2067-MBS (Mobile Broadband System), ACTS AC230-SAMBA (System for Advanced Mobile Broadband Applications), and ESA/ESTEC-ILASH (Integrated Lens Antenna Shaping). He has coauthored a book, a book chapter, more than 140 peer-reviewed technical papers in international journals and conference proceedings, and several patents in the areas of antennas and radio wave propagation modeling. His current research interests include dielectric antennas for millimeter-wave applications, antennas and propagation modeling for personal communication systems, RFID antennas, artificial dielectrics, and metamaterials.

Prof. Fernandes was a Co-Guest Editor of the special issue on "Antennas and Propagation at Millimeter and Submillimeter Waves" from the IEEE TRANSACTIONS ON ANTENNAS AND PROPAGATION.

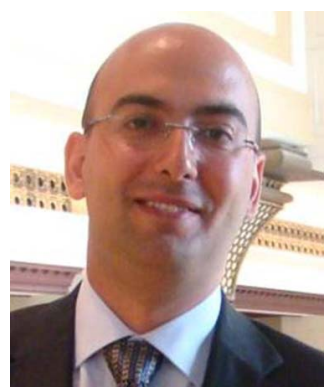

Jorge R. Costa was born in Lisbon, Portugal, in 1974. He received the Licenciado and Ph.D. degrees in electrical and computer engineering from the Technical University of Lisbon, Lisbon, in 1997 and 2002, respectively.

$\mathrm{He}$ is currently a Researcher at the Instituto de Telecomunicações, Lisbon. He is also an Associate Professor at the Departamento de Ciências e Tecnologias da Informação, Instituto Universitário de Lisboa (ISCTE-IUL). He is the coauthor of four patent applications and more than 100 contributions to peer-reviewed journals and international conference proceedings. More than twenty of those papers have appeared in IEEE journals. His current research interests include lenses, reconfigurable antennas, MEMS switches, UWB, MIMO, and RFID antennas.

Prof. Costa is currently serving as an Associate Editor of the IEEE TRANSACTIONS ON ANTENNAS AND PROPAGATION and was a Guest Editor of the special issue on "Antennas and Propagation at Millimeter and Submillimeter Waves" from the same journal. 


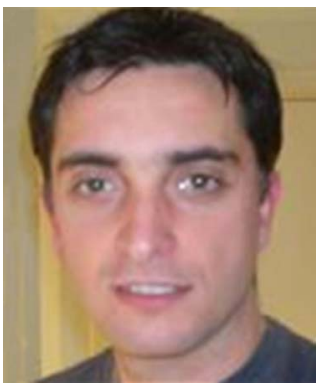

Eduardo B. Lima received the Licenciado and M.Sc. degrees in electrical and computer engineering in 2003 and 2008, respectively, from the Technical University of Lisbon, Lisbon, Portugal, where he is currently working toward the Ph.D. degree in electrical and computer engineering in the Instituto Superior Técnico.

In 2004, he joined the Instituto de Telecomunicações, Lisbon, where he is a Research Laboratory Engineer. He is the coauthor of one patent application and more than 30 technical papers in international journals and conference proceedings in the area of antennas, one of which was awarded the CST University Publication Award 2010. His current research interests include dielectric lens antennas, Fabry-Pérot cavity antennas, reflectors, and transmitarrays.

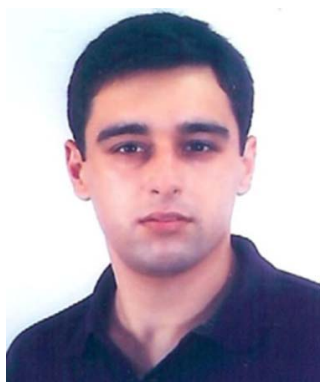

Mário G. Silveirinha received the Licenciado degree in electrical engineering from the University of Coimbra, Coimbra, Portugal, in 1998 and the Ph.D. degree in electrical and computer engineering from the Technical University of Lisbon, Lisbon, Portugal, in 2003.

$\mathrm{He}$ is currently an Associate Professor at the Department of Electrical and Computer Engineering, University of Coimbra. His research interests include electromagnetic metamaterials, plasmonics, nano-optics, and nanophotonics. $4 \frac{0}{\sqrt{s}}$ 